\title{
On the maximum number of integer colourings with forbidden monochromatic sums
}

\author{
Hong Liu* \\ Mathematics Institute \\ University of Warwick \\ Coventry, U.K. \\ h.liu.9@warwick.ac.uk
}

\author{
Maryam Sharifzadeh ${ }^{\dagger}$ \\ Department of Mathematics and Mathematical Statistics \\ Umeå University \\ Umeå, Sweden \\ maryam.sharifzadeh@umu.se
}

Submitted: June 26, 2019; Accepted: Jan 20, 2021; Published: Mar 26, 2021

(c) The authors. Released under the CC BY-ND license (International 4.0).

\begin{abstract}
Let $f(n, r)$ denote the maximum number of colourings of $A \subseteq\{1, \ldots, n\}$ with $r$ colours such that each colour class is sum-free. Here, a sum is a subset $\{x, y, z\}$ such that $x+y=z$. We show that $f(n, 2)=2^{\lceil n / 2\rceil}$, and describe the extremal subsets. Further, using linear optimisation, we asymptotically determine the logarithm of $f(n, r)$ for $r \leqslant 5$. Similar results were obtained by Hán and Jiménez in the setting of finite abelian groups.
\end{abstract}

Mathematics Subject Classifications: 11B75,11B13

\section{Introduction and results}

A recent trend in combinatorial number theory has been to consider versions of classical problems from extremal graph theory in the sum-free setting. We state some examples. The famous theorem of Mantel from 1907 [30] states that every $n$-vertex graph with more than $\left\lfloor n^{2} / 4\right\rfloor$ edges necessarily contains a triangle. On the other hand, it is not hard to

\footnotetext{
*Supported by UK Research and Innovation Future Leaders Fellowship MR/S016325/1.

†Supported by the European Unions Horizon 2020 research and innovation programme under the Marie Curie Individual Fellowship agreement No 752426.
} 
show that every subset $A$ of $[n]:=\{1, \ldots, n\}$ of size more than $\lceil n / 2\rceil$ necessarily contains a Schur triple, or sum; that is, a triple $\{x, y, z\}$ of not necessarily distinct elements such that $x+y=z$. The name dates back to a result of Schur from 1916 which states that every $r$-colouring of $[n]$ contains yields a monochromatic Schur triple when $n$ is sufficiently large. Its graph-theoretic counterpart is Ramsey's theorem from 1928 which guarantees a monochromatic clique in any $r$-edge-colouring of a sufficiently large complete graph. The triangle removal lemma of Ruzsa and Szemerédi [33] states that every $n$-vertex graph containing $o\left(n^{3}\right)$ triangles can be made triangle-free by removing $o\left(n^{2}\right)$ edges. In the sum-free setting, the removal lemma of Green [15], and Král', Serra and Vena [27] states that every $A \subseteq[n]$ containing $o\left(n^{2}\right)$ Schur triples can be made sum-free by removing $o(n)$ elements. Erdős, Kleitman and Rothschild [12] proved that the number of $n$-vertex triangle-free graphs is $2^{n^{2} / 4+o\left(n^{2}\right)}$, that is, the obvious lower bound of taking every subgraph of a maximal triangle-free graph is, in a sense, tight. In the sum-free setting, resolving a conjecture of Cameron and Erdös [7], Green [14] and independently Sapozhenko [34] proved that, for every $i=0,1$, there exists a constant $C_{i}$, depending only on the parity of $n$, such that $[n]$ contains $\left(C_{i}+o(1)\right) 2^{n / 2}$ sum-free sets. So again, the obvious lower bound is tight.

\subsection{The Erdös-Rothschild problem for cliques in graphs}

Taking inspiration from the extremal graph theory literature, in this paper we consider another classical graph problem in the sum-free setting: the problem of Erdös and Rothschild $[10,11]$, which is stated as follows. Given an $n$-vertex graph $G$ and positive integers $r, k$, say that a colouring of its edges with $r$ colours (an $r$-edge-colouring) is valid if there are no monochromatic copies of $K_{k}$. Among all such graphs $G$, what is the maximum number $F(n, r, k)$ of valid colourings? Ramsey's theorem implies that any graph containing a sufficiently large clique has no valid colourings. Clearly, any colouring of a $K_{k}$-free graph is valid. Turán's theorem implies that the largest such graph is $T_{k-1}(n)$, the complete balanced $(k-1)$-partite graph. Thus we obtain the bound

$$
F(n, r, k) \geqslant r^{t_{k-1}(n)},
$$

where $t_{k-1}(n)$ is the number of edges in $T_{k-1}(n)$. Erdös and Rothschild conjectured that this trivial lower bound is tight when $n$ is large and $(r, k)=(2,3)$ (i.e. one uses two colours and forbids monochromatic triangles), and further, that $T_{2}(n)$ is the unique extremal graph. This was confirmed for all $n \geqslant 6$ by Yuster [38], who himself extended the conjecture to larger cliques in the case $r=2$. This was in turn verified by Alon, Balogh, Keevash and Sudakov [1], showing that for $r \in\{2,3\}$, equality holds in (1.1), and $T_{k-1}(n)$ is the unique extremal graph. They further showed that for all $r, k \in \mathbb{N}$ such that $k \geqslant 3$ and $r \geqslant 2$, the $\operatorname{limit}_{\lim _{n \rightarrow \infty}} \log _{r} F(n, r, k) /\left(\begin{array}{l}n \\ 2\end{array}\right)$ exists. In other words, there is some $h(r, k)$ such that

$$
F(n, r, k)=r^{h(r, k)\left(\begin{array}{l}
n \\
2
\end{array}\right)+o\left(n^{2}\right)} .
$$

However, for every other choice of $(r, k)$, there exists a constant $c=c(r, k)$ such that $h(r, k)>(k-2) /(k-1)+c$. Note that the edge density of $T_{k-1}(n)$ tends to $(k-2) /(k-1)$ 
with $n$, so this says that the trivial lower bound in (1.1) is not correct for all other choices of $(r, k)$. Observe that this implies that extremal graphs must therefore contain many copies of the forbidden $K_{k}$. The authors were, however, able to determine $h(3,4)$ and $h(4,4)$. The exact results in these cases were obtained by Pikhurko and Yilma [32], who showed that the unique extremal graphs for $F(n, 3,4)$ and $F(n, 4,4)$ are $T_{4}(n)$ and $T_{9}(n)$ respectively.

The problem remains unresolved, even asymptotically in the logarithmic, in all other cases. In particular, there is no (approximate) solution when the number of colours $r$ is at least five, for any $k \geqslant 3$. A generalisation of the Erdös-Rothschild problem was considered by Pikhurko, Yilma and the third author of this paper [31], wherein one may forbid differently-sized cliques for different colours. A special case of the main result is that there is a certain finite optimisation problem whose maximum is equal to $h(r, k)$. Roughly speaking, they showed that to determine $F(n, r, k)$ up to an error in the exponent, one should maximise over all possible 'layerings' of 'small' $K_{k}$-free graphs.

\subsection{The Erdős-Rothschild problem in the sum-free setting: our results}

As stated above, the purpose of this paper is to initiate the study of the Erdös-Rothschild problem in the case when our underlying discrete structure is not $K_{n}$ but $[n]$, and the forbidden substructure is not a triangle but a Schur triple. Let us formulate the problem precisely.

Problem 1.1. Given positive integers $n, r$, determine $f(n, r)$, defined as follows. For each $A \subseteq[n]$, say that a colouring $\sigma: A \rightarrow[r]$ of $A$ with $r$ colours is valid if it contains no monochromatic sums. In other words, $\sigma^{-1}(c)$ is a sum-free set for each colour $c \in[r]$. Let $f(A, r)$ be the number of valid colourings of $A$ and let

$$
f(n, r):=\max _{A \subseteq[n]} f(A, r) .
$$

Notice that Schur's theorem says that $f([n], r)=0$ whenever $n$ is sufficiently large (as a function of $r$ ). Before stating our results, let us see what we might conjecture via analogy with the graph setting. Here, for $r \in\{2,3\}$, the unique extremal graph was the largest triangle-free graph. It is not hard to see that every largest sum-free sets in $[n]$ has size at most $\lceil n / 2\rceil$, and the only subsets attaining this bound are

(i) $\mathrm{O}:=\{1,3,5, \ldots, 2\lceil n / 2\rceil-1\}$ and

(ii) $I_{2}:=\{\lfloor n / 2\rfloor+1,\lfloor n / 2\rfloor+2, \ldots, n\}$.

Additionally, if $n$ is even, the set $\{n / 2, \ldots, n-1\}$ is also a largest sum-free subset.

Our first theorem is an exact result for two colours, which states that the largest sum-free subsets of $[n]$ are also extremal subsets for Problem 1.1. 
Theorem 1.2. There exists $n_{0}>0$ such that for all integers $n \geqslant n_{0}$, we have

$$
f(n, 2)=2^{\lceil n / 2\rceil} .
$$

Moreover, the only extremal subsets are $\mathrm{O}, \mathrm{I}_{2}$; and if $n$ is even, we additionally have $\{n / 2, \ldots, n-1\},\{n / 2, \ldots, n\}$.

Note that in the sum-free setting with two colours, there is an extremal subset which is not sum-free, namely $\{n / 2, \ldots, n\}$, containing at most one sum. In the graph setting with two colours, there is a unique extremal graph, namely $T_{2}(n)$, which is $K_{3}$-free.

As in the graph setting, our results decrease in strength as the number of colours increases. When $r=3$ we can obtain the following stability theorem.

Theorem 1.3. For all positive integers n, we have

$$
f(n, 3)=3^{n / 2+o(n)} .
$$

Moreover, the following holds. For all $\varepsilon>0$ there exist $\delta, n_{0}>0$ such that for all integers $n \geqslant n_{0}$, whenever $A \subseteq[n]$ satisfies $f(A, 3) \geqslant f(n, 3) \cdot 2^{-\delta n}$, we have that either $|A \triangle \mathrm{O}| \leqslant \varepsilon n ;$ or $\left|A \triangle I_{2}\right| \leqslant \varepsilon n$.

(Here, $\triangle$ denotes symmetric difference; see Section 2.1.) Finally, we asymptotically determine the logarithm of $f(n, r)$ when $r \in\{4,5\}$.

Theorem 1.4. For $r \in\{4,5\}$ and all positive integers $n$, we have

$$
f(n, r)=\left(r\left\lfloor\frac{r^{2}}{4}\right\rfloor\right)^{\frac{n}{4}+o(n)} .
$$

In particular, we are able to asymptotically solve the 5-colour case of the ErdösRothschild problem in the sum-free setting, in contrast to the graph setting in which it is wide open for triangles and larger cliques. (This turns out to be a consequence of the rigid structure of large maximal sum-free sets.) The (asymptotic) lower bounds in Theorems 1.2-1.4 come from the facts that

$$
f(\mathrm{O}, r)=f\left(I_{2}, r\right)=r^{\lceil n / 2\rceil} \quad \text { and } \quad f\left(\mathrm{O} \cup I_{2}, r\right) \geqslant\left(r\left\lfloor\frac{r^{2}}{4}\right\rfloor\right)^{\left\lfloor\frac{n}{4}\right\rfloor} .
$$

The first assertion follows from the fact that every $r$-colouring of a sum-free set $A$ is valid, so $f(A, r)=r^{|A|}$. For the second, note that any colouring $\sigma: \mathrm{O} \cup I_{2} \rightarrow[r]$ with $\sigma(x) \in\{1, \ldots,\lfloor r / 2\rfloor\}$ whenever $x \in O \backslash I_{2}$, and $\sigma(y) \in\{\lfloor r / 2\rfloor+1, \ldots, r\}$ whenever $y \in I_{2} \backslash O$ (and $O \cap I_{2}$ coloured arbitrarily), is valid, giving the claimed bound.

Note that $r^{1 / 2}>\left(r\left\lfloor r^{2} / 4\right\rfloor\right)^{1 / 4}$ for $r \in\{2,3\}$, while this inequality becomes an equality for $r=4$, and reverses for $r \geqslant 5$. It is tempting to believe that the bound in Theorem 1.4 holds for all $r \geqslant 4$. However, this is not true. Indeed, when $r$ is large, the set $A:=$ $\mathrm{O} \cup I_{2} \cup\{x \in[n]: x \equiv 1,4 \bmod 5\}$ contains exponentially more valid $r$-colourings than 
$\mathrm{O} \cup I_{2}$. To see this, suppose for simplicity that $3 \mid r$, and partition the colours $[r]$ into three sets $\mathcal{C}_{\mathrm{O}}, \mathcal{C}_{I_{2}}, \mathcal{C}_{1,4}$ of equal size. Let any colour in $\mathcal{C}_{\mathrm{O}}$ be allowed for any $x \in \mathrm{O}$, and do the same for $\mathcal{C}_{I_{2}}$ and $I_{2}$, and for $\mathcal{C}_{1,4}$ and $\{x \equiv 1,4 \bmod 5\}$. So, for example the largest $10 t+1$ which is less than $n$ has all $r$ colours allowed since it lies in all three sets. Any colouring which only uses allowed colours for each vertex is valid, since each of the three sets is sum-free. Calculating the sizes of the intersections, we see that

$$
f(n, r) \geqslant\left(\frac{r}{3}\right)^{\frac{8 n}{20}}\left(\frac{2 r}{3}\right)^{\frac{7 n}{20}} r^{\frac{2 n}{20}} \cdot r^{o(n)} \geqslant r^{\frac{17 n}{20}+o(n)} .
$$

It would also be interesting to see if for every fixed integer $r \geqslant 6$, the limit

$$
\lim _{n \rightarrow \infty} \log f(n, r) / n
$$

exists.

\subsection{Some remarks on the methods and proofs}

An important tool in our proof is the Green's container theorem for finite abelian groups (Theorem 2.2). The special case that we need states that, for every positive integer $n$, there is a small family $\mathcal{F}$ of subsets of $[n]$, called containers, each of which is almost sum-free, and such that every sum-free subset of $[n]$ lies in some member of $\mathcal{F}$.

In the proof of the main result in [1] and other Erdös-Rothschild-type results for graphs, Szemerédi's regularity lemma [36] is used to approximate a large graph $G$ by another graph of bounded size (the reduced graph). Then, for each valid $r$-edge-colouring $\sigma$ of $G$, for each $i \in[r]$ one can approximate the $K_{k}$-free subgraph $\sigma^{-1}(i)$ of $G$ by a $K_{k}$-free graph of bounded size. In our proofs, for each valid $r$-colouring of $[n]$, we approximate the sum-free subset $\sigma^{-1}(i)$ of $[n]$ by a container.

We use Theorem 2.2 to reduce the problem of determining $f(n, r)$ to solving an optimisation problem (Problem 2) whose maximum approaches $\log f(n, r) / n$ as $n$ tends to infinity (Theorem 3.3). Roughly speaking, Problem 2 involves layering sum-free subsets $A_{1}, \ldots, A_{r}$ of $[n]$ and measuring a weighted overlap $g\left(A_{1}, \ldots, A_{r}\right)$.

To attack Problem 2, we require a second important tool, namely a very strong stability theorem of Deshoulliers, Freiman, Sós, and Temkin [9], which was recently strengthened by Tran [37]. This states that every sum-free subset of $[n]$ is either 'small', or has a very rigid structure: either it contains only odd elements, or it somehow resembles the interval $I_{2}$. Now it turns out that, if $r$ is small and $\left(A_{1}, \ldots, A_{r}\right)$ is a maximiser for Problem 2, then at most one of the $A_{i}$ can be small. The rigid structure of the others means that the feasible set for Problem 2 is not too large. In fact, for $r \in\{2,3\}$, it can be easily solved at this stage, and we find that either all of $A_{1}, \ldots, A_{r}$ are close to $\mathrm{O}$; or they are all close to $I_{2}$. This proves Theorem 1.3 and completes the first step of the proof of Theorem 1.2.

To complete the proof of Theorem 1.2, we have two cases to consider. The solution to Problem 2 when $r=2$ implies that any subset $A \subseteq[n]$ with $f(A, r)=f(n, r)$ satisfies either (1) $|A \triangle \mathrm{O}|=o(n)$; or (2) $\left|A \triangle I_{2}\right|=o(n)$. We use stability arguments, together with techniques from [5], to obtain the exact structure of $A$. 
For $r \in\{4,5\}$, we find a reduction of Problem 2 to a linear optimisation problem. First, for each $i \in[r]$, and any feasible $\left(A_{1}, \ldots, A_{r}\right) \subseteq[n]^{r}$, we obtain $d_{i} \in[0,1]$ which are each functions of $A_{1}, \ldots, A_{r}$ and $n$ and such that $g\left(A_{1}, \ldots, A_{r}\right)$ is linear in $d_{1}, \ldots, d_{r}$. Now, using the structural information returned from stability, we obtain constraints, linear in $d_{1}, \ldots, d_{r}$, which every maximiser $\left(A_{1}, \ldots, A_{r}\right)$ must satisfy. This gives rise to a linear program in the variables $d_{1}, \ldots, d_{r}$. Now, this linear program is a relaxation of Problem 2 , so its maximiser may not correspond to a feasible solution $\left(A_{1}, \ldots, A_{r}\right)$ of Problem 2. But, if we can exhibit a feasible tuple $\left(A_{1}, \ldots, A_{r}\right)$ such that the maximum $M$ of this program satisfies $M=g\left(A_{1}, \ldots, A_{r}\right)$, then $\left(A_{1}, \ldots, A_{r}\right)$ is a maximiser of Problem 2. Thus our task is to find enough constraints (of sufficient strength) so that this is possible. In so doing, we will prove Theorem 1.4.

\subsection{The Erdös-Rothschild problem in other settings}

Erdős and Rothschild also considered the problem of counting monochromatic $H$-free colourings, for an arbitrary fixed graph $H$. In [1], it is shown that the analogue of their main result for cliques in fact holds when $H$ is colour-critical. Further cases including matchings, stars, paths, trees were investigated in [20, 21]. Other works have considered a fixed forbidden colour pattern of $H$, see $[3,6,22,24]$.

An analogous problem for directed graphs was solved by Alon and Yuster [2], who determined, for each $k$-vertex tournament $T$, the maximum number of $T$-free orientations of an $n$-vertex graph, when $n$ is sufficiently large. The hypergraph analogue was addressed in $[19,28,29]$.

The authors of [8] and [19] considered the problem of counting the number of colourings of families of $r$-sets such that every colour class is $\ell$-intersecting. A related result in the context of vector spaces over a finite field $G F(q)$ is proved in [23]. These results are Erdös-Rothschild versions of the classical Erdös-Ko-Rado theorem.

During the preparation of this paper, we became aware of the results of Hàn and Jiménez [18] who recently studied similar questions in the setting of finite abelian groups. Given a finite abelian group $(\Gamma,+)$, define an $r$-colouring of $A \subseteq \Gamma$ to be valid if it has no monochromatic sum. Let $f(\Gamma, r)$ be the maximum number of valid $r$-colourings among all subsets $A$ of $\Gamma$. The results of Hàn and Jiménez show a close relationship between $f(\Gamma, r)$ and the largest sum-free sets of $\Gamma$, and characterise for $r \leqslant 5$ the extremal sets. As in [18], our proof begins with an application of the container method. But, in the setting of abelian groups, there is much more structure than in the integer setting, and thus one can obtain much stronger stability, leading to precise results for larger values of $r$ than those obtained here. Most of the work in this paper goes into dealing with 'interval-like' sets, (an analogue of which) does not appear in the abelian group setting. We remark that Hàn and Jiménez's result and ours do not imply one another.

\subsection{Organisation of the paper}

Section 2 sets up the notation we will use and contains the statements of results on sumfree sets necessary for the proof. In Section 3 we define Problem 2, the optimisation 
problem whose maximum is a parameter $g(n, r)$ which is closely related to $f(n, r)$. Then in Sections 4, 5 and 6 we prove Theorems 1.3, 1.2 and 1.4 respectively. We make some concluding remarks in Section 7.

\section{Notation and Preliminaries}

In this section we define the notation that we will use, and some results on sum-free subsets which are needed in our proofs.

\subsection{Notation}

Given integers $m, n$ such that $m \leqslant n$, we write $[m, n]$ to denote the set $\{m, \ldots, n\}$, and write $[n]:=[1, n]$. For a set $A \subseteq[n]$, we define $d(A)=|A| / n$ and $\min (A)$ to be the density and the minimum element of $A$, respectively. We also define $E$ and $\mathrm{O}$ to be respectively the set of all even and odd integers in $[n]$. As we defined earlier, $I_{2}:=[\lfloor n / 2\rfloor+1, n]$ and $I_{1}:=[\lfloor n / 2\rfloor]$ (we suppress the dependence on $n$ in the notation). Given $A, B \subseteq \mathbb{Z}$, we write $A+B:=\{a+b: a \in A, b \in B\}$, and $A \triangle B:=(A \backslash B) \cup(B \backslash A)$ denotes the symmetric difference of $A$ and $B$. For any $x \in \mathbb{Z}$, we also write $x \cdot A:=\{x a: a \in A\}$. Logarithms will always be taken to the base 2 .

\subsection{Tools for sum-free subsets}

The first result we state is a very strong stability theorem for sum-free subsets due to Deshouillers, Freiman, Temkin and Sós [9]. It states that every large sum-free set $S \subseteq[n]$ either contains no even number, or is, in a certain sense, close to the interval $I_{2}$.

Theorem 2.1 ([9]). Every sum-free set $S$ in $[n]$ satisfies at least one of the following conditions:

(a) $|S| \leqslant 2 n / 5+1 ;$

(b) $S$ consists of odd numbers;

(c) $|S| \leqslant \min (S)$.

Throughout the rest of the paper, we refer to such sum-free sets as type (a), type (b), and type (c) respectively.

We use the following container theorem of Green [15] (see also Green-Ruzsa [16, 17]), which, for large $n$, guarantees a small collection of subsets of $[n]$ which somehow approximates the collection of sum-free sets. We should also mention that (hyper)graph containers have been used successfully in many contexts, see [4, 25, 26, 35].

Theorem 2.2 (Proposition 6 in [14]). For all $\varepsilon>0$ there exists $n_{0}>0$ such that, for all integers $n \geqslant n_{0}$, there exists a family $\mathcal{F}$ of subsets of $[n]$ with the following properties:

(i) Every $F \in \mathcal{F}$ contains at most $\varepsilon n^{2}$ Schur triples; 
(ii) If $S \subseteq[n]$ is sum-free, then $S \subseteq F$ for some $F \in \mathcal{F}$;

(iii) $|\mathcal{F}| \leqslant 2^{\varepsilon n}$;

(iv) $|F| \leqslant(1 / 2+\varepsilon) n$ for all $F \in \mathcal{F}$.

It is convenient for us to include property (iv) although it is not explicitly stated in [14], but follows immediately from (i), Theorem 2.1 and Theorem 2.3. Given a sumfree set $S \subseteq[n]$, the set $F \in \mathcal{F}$ guaranteed by (ii) is called a container for $S$. We also need the following removal lemma of Green [14, 15], and Král', Serra and Vena [27], which guarantees that a subset of $[n]$ containing $o\left(n^{2}\right)$ sums can be made sum-free by removing $o(n)$ elements.

Theorem 2.3 ([14, 15, 27]). For all $\varepsilon>0$, there exists $\delta, n_{0}>0$ such that the following holds for all integers $n \geqslant n_{0}$. Suppose that $A \subseteq[n]$ is a set containing at most $\delta n^{2}$ Schur triples. Then there exist $B, C \subseteq[n]$ such that $A=B \cup C$ where $B$ is sum-free and $|C| \leqslant \varepsilon n$.

This result comes from applying a regularity lemma and consequently the dependence of $\delta$ on $\varepsilon$ is rather poor: $1 / \delta$ is at most a tower of twos of height logarithmic in $1 / \varepsilon$, which follows from [27] and [13]. Finally, we will use the following folklore inequality which bounds the size of the set $A+B$ :

Proposition 2.4. For all finite non-empty subsets $A, B$ of $\mathbb{Z}$, we have that $|A+B| \geqslant$ $|A|+|B|-1$.

Proof. Write $A:=\left\{a_{1}<\cdots<a_{k}\right\}$ and $B:=\left\{b_{1}<\cdots<b_{\ell}\right\}$. Then $A+B \supseteq\left\{a_{1}+b_{1}<\right.$ $\left.a_{1}+b_{2}<\cdots<a_{1}+b_{\ell}<a_{2}+b_{\ell}<a_{3}+b_{\ell}<\cdots<a_{k}+b_{\ell}\right\}$.

\section{An equivalent covering problem}

In this section, we define a new maximisation problem whose value $g(n, r)$ is closely related to $f(n, r)$. Then, for the rest of the paper, it suffices to consider this new problem. To motivate the problem, consider the following procedure for finding a subset $A \subseteq[n]$ with many valid colourings. Let $r \in \mathbb{N}$ be the number of colours, as usual, and choose sum-free subsets $A_{1}, \ldots, A_{r}$ of $[n]$. Then the number of valid colourings of $\bigcup_{i \in[r]} A_{i}$ is at least the number of colourings $\sigma$ which colours $x$ with some $i$ such that $x \in A_{i}$. If $x$ lies in many $A_{i}$ then the number of choices for $\sigma(x)$ is large. So a choice of $A_{1}, \ldots, A_{r}$ with a large appropriately weighted overlap generates many valid colourings. We now make this precise.

Problem 3.1. Given $n, r \in \mathbb{N}$, determine $g(n, r)$, defined as follows. Given a tuple $\left(A_{1}, \ldots, A_{r}\right)$ of sum-free subsets of $[n]$, for each $I \in 2^{[r]}$, let $E_{I}:=\bigcap_{i \in I} A_{i} \backslash \bigcup_{j \notin I} A_{j}$ be the set of $x \in[n]$ which lie in $A_{i}$ if and only if $i \in I$. Define

$$
g\left(A_{1}, \ldots, A_{r}\right):=\frac{1}{n} \sum_{I \in 2^{[r]} \backslash\{\emptyset\}}\left|E_{I}\right| \log |I| .
$$


Equivalently, for each $i \in[r]$ let $D_{i}:=\bigcup_{I \in 2^{[r]:|I|=i}} E_{I}$; that is, the set of all elements that are in exactly $i$ different $A_{j}$ 's. Let $d_{i}:=\left|D_{i}\right| / n$ and define $\left(d_{1}, \ldots, d_{r}\right)$ to be the intersection vector of $\left(A_{1}, \ldots, A_{r}\right)$. Let $d_{0}:=1-\sum_{i \in[r]} d_{i}$. Then

$$
g\left(A_{1}, \ldots, A_{r}\right)=\sum_{i \in[r]} d_{i} \log i
$$

Define

$$
g(n, r):=\max \left\{g\left(A_{1}, \ldots, A_{r}\right): A_{i} \subseteq[n] \text { is sum-free for all } i \in[r]\right\} .
$$

Define also $g(A, r)$ to be the maximum of $g\left(A_{1}, \ldots, A_{r}\right)$ over all tuples of sum-free subsets of $[n]$ such that $\bigcup_{i \in[r]} A_{i}=A$.

Remark 3.2. It is not hard to see that, for every $n$ and $r$, there is always some tuple $\left(A_{1}, \ldots, A_{r}\right)$ of sum-free subsets of $[n]$ which is extremal (that is, $\left.g\left(A_{1}, \ldots, A_{r}\right)=g(n, r)\right)$, and $A_{i}$ is a maximal sum-free subset for all $i \in[r]$. It will be useful to choose such an extremal tuple later, since if we know e.g. that $A_{i}$ contains no even element, then by Theorem 2.1 we can assume that $A_{i}=\mathrm{O}$.

The first step in the proofs of Theorems $1.2-1.4$ is to show that these problems are, in a sense, equivalent.

Theorem 3.3. For all $\varepsilon>0$ and $r \in \mathbb{N}$, there exists $n_{0}>0$ such that the following holds for all integers $n \geqslant n_{0}$. Let $A \subseteq[n]$. Then there exists $A^{\prime} \subseteq A$ with $\left|A^{\prime}\right| \geqslant|A|-$ en for which

$$
2^{g(A, r) n} \leqslant f(A, r) \leqslant 2^{\left(g\left(A^{\prime}, r\right)+\varepsilon\right) n} .
$$

Therefore

$$
g(n, r) \leqslant \frac{\log f(n, r)}{n} \leqslant g(n, r)+\varepsilon
$$

Proof. We first prove the lower bound (for all $n$ and $r$ ). Fix integers $n, r$ and let $A \subseteq[n]$. Choose a tuple $\left(A_{1}, \ldots, A_{r}\right)$ of sum-free subsets of $[n]$ whose intersection vector $\left(d_{1}, \ldots, d_{r}\right)$ is extremal, i.e. satisfies

$$
\sum_{i \in[r]} d_{i} \log i=g\left(A_{1}, \ldots, A_{r}\right)=g(A, r)
$$

For each $I \in 2^{[r]} \backslash\{\emptyset\}$, define $E_{I}$ as in the statement of Problem 3.1. Consider any colouring $\sigma: A \rightarrow[r]$ such that, for each $I \in 2^{[r]} \backslash\{\emptyset\}$ and $x \in E_{I}$, we have $\sigma(x) \in I$. Then $\sigma^{-1}(i) \subseteq A_{i}$ for all $i \in[r]$, so the fact that $A_{i}$ is sum-free for all $i \in[r]$ implies that $\sigma$ is valid. Thus the number of such $\sigma$ is a lower bound for the total number of valid colourings, and so

$$
f(A, r) \geqslant \prod_{I \in 2^{[r]} \backslash\{\emptyset\}}|I|^{\left|E_{I}\right|}=\prod_{i \in[r]} i^{d_{i} n} \stackrel{(3.3)}{=} 2^{g(A, r) n},
$$


as required.

For the remainder of the proof we focus on the upper bound. Fix an integer $r$ and let $\varepsilon>0$. We may assume that $\varepsilon \ll 1 / r$. Choose $\eta$ such that $0<\eta \ll \varepsilon$. Apply Theorem 2.3 to obtain $\gamma, n_{0}>0$ such that, for all integers $n \geqslant n_{0}$, every $A \subseteq[n]$ which contains at most $\gamma n^{2}$ Schur triples may be made sum-free by removing at most $\eta n$ elements. Without loss of generality we may assume that $\gamma \ll \eta$. Theorem 2.2 implies that, by increasing $n_{0}$ if necessary, for all integers $n \geqslant n_{0}$, there exists a family $\mathcal{F}=\mathcal{F}_{n}$ of containers such that

(i) every $F \in \mathcal{F}$ contains at most $\gamma n^{2}$ Schur triples;

(ii) every sum-free subset of $[n]$ lies in at least one $F \in \mathcal{F}$;

(iii) $|\mathcal{F}| \leqslant 2^{\gamma n}$; and

(iv) $|F| \leqslant(1 / 2+\gamma) n$ for all $F \in \mathcal{F}$.

Without loss of generality, we may assume that $1 / n_{0} \ll \gamma$ and $\varepsilon \ll 1$. We have the hierarchy

$$
1 / n_{0} \ll \gamma \ll \eta \ll \varepsilon \ll 1 .
$$

Given any $n \geqslant n_{0}$ and the family $\mathcal{F}$ of containers, for each $F \in \mathcal{F}$, fix a largest sum-free subset $F^{*}$ of $F$ (note that there may be more than one choice of $F^{*}$, but we just pick one of these). Then (i) together with Theorem 2.3 implies that $|F|-\eta n \leqslant\left|F^{*}\right| \leqslant|F|$.

Now let $n \geqslant n_{0}$ be an integer, and $A \subseteq[n]$ be arbitrary. Consider any fixed valid $r$-colouring $\sigma$ of $A$. Then $\sigma^{-1}(i)$ is sum-free for all $i \in[r]$. By (ii), we may choose a tuple $\left(F_{1}, \ldots, F_{r}\right) \in \mathcal{F}^{r}$ of containers such that $\sigma^{-1}(i) \subseteq F_{i}$. By (i), $F_{i}$ contains at most $\gamma n^{2}$ Schur triples for all $i \in[r]$. For each $i \in[r]$, let us write $F_{i}^{*}:=\left(F_{i}\right)^{*}$ for the largest sumfree subset of $F_{i}$ we fixed earlier. Then $\left|F_{i}^{*}\right| \geqslant\left|F_{i}\right|-\eta n$. Thus, for each valid colouring $\sigma: A \rightarrow[r]$, we obtain a tuple $\left(F_{1}^{*}, \ldots, F_{r}^{*}\right)$. Observe that

$$
\left|\sigma^{-1}(i) \backslash F_{i}^{*}\right| \leqslant \eta n
$$

but $F_{i}^{*}$ may contain many elements which do not lie in $\sigma^{-1}(i)$.

We now claim that the following procedure generates every valid colouring $\sigma$ of $A$, and therefore the number of choices in this procedure is an upper bound on $f(A, r)$. Each choice will generate a colouring $\tau$ :

1. For all $i \in[r]$, choose a container $G_{i} \in \mathcal{F}$, and let $G_{i}^{*}$ be the largest sum-free subset of $G_{i}$ we fixed earlier.

2. For each $I \in 2^{[r]} \backslash\{\emptyset\}$, let

$$
E_{I}^{\prime}:=\left(\bigcap_{i \in I} G_{i}^{*} \backslash \bigcup_{j \notin I} G_{j}^{*}\right) \cap A .
$$

Let also $D_{i}^{\prime}:=\bigcup_{I \subseteq[r]:|I|=i} E_{I}^{\prime}$ for each $i \in[r]$. So $D_{i}^{\prime}$ is the set of those elements in $A$ which lie in exactly $i$ of the $G_{j}^{*}$. Let $d_{i}^{\prime}:=\left|D_{i}^{\prime}\right| / n$ for all $i \in[r]$. 
3. For each $I \in 2^{[r]} \backslash\{\emptyset\}$ and $x \in E_{I}^{\prime}$, choose $i \in I$ and set $\tau(x):=i$.

4. For each uncoloured $y \in A$, let $\tau(y) \in[r]$ be arbitrary.

We need to show that there is a choice in (1)-(4) which will yield $\tau=\sigma$. In (1), for each $i \in[r]$, (ii) and the fact that $\sigma$ is valid implies that we can choose $G_{i}:=F_{i} \in \mathcal{F}$ such that $\sigma^{-1}(i) \subseteq F_{i}$. Note that $G_{i}^{*}=F_{i}^{*}$ for all $i \in[r]$. The choice in (2) is fixed by our choices in (1). In (3), by construction, for every $x \in\left(\bigcup_{i \in[r]} F_{i}^{*}\right) \cap A$, we have that $x \in E_{I}^{\prime}$ for some $I \ni \sigma(x)$. Thus for every $x \in\left(\bigcup_{i \in[r]} F_{i}^{*}\right) \cap A$ we can choose $\tau(x):=\sigma(x)$. In (4) we are free to colour the uncoloured elements of $A$ with $\sigma$. Since $\sigma$ was an arbitrary valid colouring of $A$, we have proved the claim.

Thus it remains to count the number of colourings generated by (1)-(4). Given a tuple $\left(G_{1}, \ldots, G_{r}\right) \in \mathcal{F}^{r}$ of containers, let $\mathcal{C}$ be the set of colourings $\tau: A \rightarrow[r]$ generated by it, i.e. the set of $\tau$ which arise from the procedure after fixing the choice $\left(G_{1}, \ldots, G_{r}\right)$ in $(1)$. Observe that $\left(G_{1}, \ldots, G_{r}\right)$ gives rise to a unique tuple $\left(G_{1}^{*}, \ldots, G_{r}^{*}\right)$. Then, since the only choices are in (3) and (4), we have

$$
|\mathcal{C}| \leqslant \prod_{j \in[r]} j^{\left|D_{j}^{\prime}\right|} \cdot r\left|A \backslash \bigcup_{i \in[r]} G_{i}^{*}\right| \stackrel{(3.4)}{\leqslant} \prod_{j \in[r]} j^{d_{j}^{\prime} n} \cdot r^{r \eta n} .
$$

Taking logarithms, we have, bounding very loosely, that

$$
\frac{\log |\mathcal{C}|}{n} \leqslant \sum_{j \in[r]} d_{j}^{\prime} \log j+\sqrt{\eta}=g\left(G_{1}^{*} \cap A, \ldots, G_{r}^{*} \cap A\right)+\sqrt{\eta} \leqslant g\left(A^{\prime}, r\right)+\sqrt{\eta},
$$

where $A^{\prime}:=A \cap \bigcup_{i \in[r]} G_{i}^{*}$. So $\left|A^{\prime}\right| \geqslant|A|-r \eta n \geqslant|A|-\varepsilon n$. But, by (iii), the number of choices of $\left(G_{1}, \ldots, G_{r}\right) \in \mathcal{F}^{r}$ is at most $|\mathcal{F}|^{r} \leqslant 2^{r \gamma n}$, so

$$
f(A, r) \leqslant 2^{r \gamma n} \cdot 2^{g\left(A^{\prime}, r\right) n} \cdot 2^{\sqrt{\eta} n} \leqslant 2^{\left(g\left(A^{\prime}, r\right)+2 \sqrt{\eta}\right) n} \leqslant 2^{\left(g\left(A^{\prime}, r\right)+\varepsilon\right) n},
$$

completing the proof of the upper bound. The second assertion is an obvious consequence of the first.

The discussion about lower bounds after the statement of Theorem 1.4 amounts to the following inequalities: For all integers $n \geqslant r \geqslant 2$, we have

$$
\begin{gathered}
\frac{\log f(n, r)}{n} \geqslant g(n, r) \geqslant g(\underbrace{I_{2}, \ldots, I_{2}}_{r})=\frac{\lceil n / 2\rceil}{n} \cdot \log r ; \quad \text { and } \\
\frac{\log f(n, r)}{n} \geqslant g(n, r) \geqslant g(\underbrace{I_{2}, \ldots, I_{2}}_{\lfloor r / 2\rfloor}, \underbrace{\mathrm{O}, \ldots, \mathrm{O}}_{\lceil r / 2\rceil}) \geqslant \frac{\lfloor n / 4\rfloor}{n} \log \left(r\left\lfloor\frac{r^{2}}{4}\right\rfloor\right) .
\end{gathered}
$$

Figure 1 shows these three constructions in the case when $r=4$, when they each give rise to roughly the same lower bound. Theorem 1.4) implies that each one is in fact an approximate optimal solution of Problem 1. 


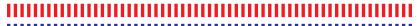
年

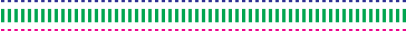
|||||||||||||||||||||||||||||||||||||||||||||||||||||||||||||||||||

1

$\left\lfloor\frac{n}{2}\right\rfloor$

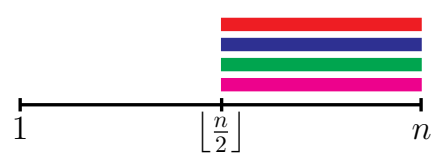

Figure 1: Three approximate solutions to Problem 2 for $r=4$.

Observe the following easy correspondence between feasible solutions of Problems 1 and 2. Given a feasible solution $A \subseteq[n]$ of Problem 1 and a valid $r$-colouring $\sigma$ of $A$, we have that $\left(\sigma^{-1}(1), \ldots, \sigma^{-1}(r)\right)$ is a feasible solution of Problem 2. Given a feasible solution $\left(A_{1}, \ldots, A_{r}\right) \subseteq[n]^{r}$ of Problem 3.1, we have that $A_{1} \cup \ldots \cup A_{r}$ is a feasible solution of Problem 1.1.

Theorem 3.3 is essentially an analogue of the main result of [31]. Recall the parameters $h(r, k)$ and $F(n, r, k)$ related to the graph problem defined in the introduction, related by (1.2). Informally speaking, determining $g(n, r)$ involves layering $r$ sum-free subsets of $[n]$ so that an appropriately weighted overlap is as large as possible, whereas determining $h(r, k)$ involves layering $r$ finite $K_{k}$-free graphs so that their weighted overlap is as large as possible. Importantly and unfortunately, $g(n, r)$ does of course depend on $n$. However, the cases in which $F(n, r, k)$ has been determined (when $r$ is small) give us some valuable intuition for determining $g(n, r)$ (and hence approximately determining $f(n, r)$ ): namely that for an extremal tuple $\left(A_{1}, \ldots, A_{r}\right)$ of sum-free sets, each $A_{i}$ should perhaps be a largest sum-free set: either $\mathrm{O}$ or $I_{2}$. Unlike in the case of graphs, our ground set $[n]$ comes with a fixed labelling. So there is only one way to layer, say, $\mathrm{O}$ and $I_{2}$, whereas there are many ways to layer any two $r$-vertex graphs $G$ and $H$.

\section{The proof of Theorem 1.3}

Given Theorem 3.3, it is now a fairly simple task to obtain stability in the case when $r=2,3$. Indeed, it suffices to prove stability for Problem 2.

Lemma 4.1. Let $r \in\{2,3\}$. For all $\varepsilon>0$, there exists $n_{0}>0$ such that the following holds for all integers $n \geqslant n_{0}$. Let $A \subseteq[n]$ be such that $g(A, r) \geqslant g(n, r)-\varepsilon /(50 r)$. Then either $|A \triangle \mathrm{O}| \leqslant \varepsilon n$ or $\left|A \triangle I_{2}\right| \leqslant \varepsilon n$.

Proof. Let $\varepsilon>0$ and assume without loss of generality that $\varepsilon<1 / 100$. Let also $n$ be a sufficiently large integer compared to $\varepsilon$. Choose a tuple $\left(A_{1}, \ldots, A_{r}\right)$ of sum-free subsets of $[n]$ such that $g\left(A_{1}, \ldots, A_{r}\right)=g(A, r)$. Let $\left(d_{1}, \ldots, d_{r}\right)$ be its intersection vector. Recall from (3.6) that $g(n, r) \geqslant(1 / 2) \cdot \log r$. So $g(A, r) \geqslant(1 / 2) \cdot \log r-\varepsilon /(50 r)$. We will need the following claim.

Claim 4.2. It suffices to show that $d_{r} \geqslant 1 / 2-\varepsilon /(3 r)$.

Proof: Let $A^{\prime}:=A_{1} \cap \ldots \cap A_{r}$. Assume that $\left|A^{\prime}\right|=d_{r} n \geqslant(1 / 2-\varepsilon /(3 r)) n$. Clearly $A^{\prime}$ is a sum-free subset of $[n]$. Suppose that $A^{\prime}$ contains at least one even element. 
Then Theorem 2.1 implies that $A^{\prime}$ is a sum-free set of type (c), so $\min \left(A^{\prime}\right) \geqslant\left|A^{\prime}\right| \geqslant$ $(1 / 2-\varepsilon /(3 r)) n$, and so $\left|A^{\prime} \triangle I_{2}\right| \leqslant 2 \varepsilon n /(3 r)$. Otherwise (if every element of $A^{\prime}$ is odd) we have $\left|A^{\prime} \triangle \mathrm{O}\right| \leqslant \varepsilon n /(3 r)$. Finally, since every sum-free subset of $[n]$ has size at most $\lceil n / 2\rceil$, we have

$$
\left|A \triangle A^{\prime}\right| \leqslant \sum_{i \in[r]}\left|A_{i} \triangle A^{\prime}\right| \leqslant r\left(\lceil n / 2\rceil-d_{r} n\right) \leqslant \varepsilon n / 3+r .
$$

So, in the first case (if $\left|A^{\prime} \triangle I_{2}\right| \leqslant 2 \varepsilon n /(3 r)$ ), we have $\left|A \triangle I_{2}\right| \leqslant\left|A \triangle A^{\prime}\right|+\left|A^{\prime} \triangle I_{2}\right| \leqslant \varepsilon n$. Similarly, in the second case, we have $|A \triangle \mathrm{O}| \leqslant \varepsilon n$.

First consider the case when $r=2$. Then

$$
\frac{1}{2}-\frac{\varepsilon}{50 r} \leqslant g(A, 2)=g\left(A_{1}, A_{2}\right)=d_{2}
$$

as required. Now let $r=3$. Then

$$
\frac{1}{2} \cdot \log 3-\frac{\varepsilon}{50 r} \leqslant g(A, 3)=g\left(A_{1}, A_{2}, A_{3}\right)=d_{2}+d_{3} \cdot \log 3 .
$$

Recall that each $A_{i}$ is sum-free with size at most $\lceil n / 2\rceil$, so

$$
d_{1}+2 d_{2}+3 d_{3}=\frac{1}{n}\left(\left|A_{1}\right|+\left|A_{2}\right|+\left|A_{3}\right|\right) \leqslant \frac{3}{n}\left\lceil\frac{n}{2}\right\rceil \leqslant \frac{3}{2}+\frac{\varepsilon}{100 r},
$$

which implies that $d_{2}+3 d_{3} / 2 \leqslant 3 / 4+\varepsilon /(200 r)$. Therefore, if $d_{3}<1 / 2-\varepsilon /(3 r)$, we have

$$
\begin{aligned}
d_{2}+d_{3} \cdot \log 3 & =d_{2}+\frac{3}{2} d_{3}+\left(\log 3-\frac{3}{2}\right) \cdot d_{3}<\frac{3}{4}+\frac{\varepsilon}{200 r}+\left(\log 3-\frac{3}{2}\right) \cdot\left(\frac{1}{2}-\frac{\varepsilon}{3 r}\right) \\
& =\frac{1}{2} \log 3-\left(\frac{\log 3}{3}-\frac{1}{200}-\frac{1}{2}\right) \cdot \frac{\varepsilon}{r}<\frac{1}{2} \log 3-\frac{\varepsilon}{50 r},
\end{aligned}
$$

a contradiction to (4.1).

Note that, in the case $r=3$, the proof proceeds by solving a linear program in variables $d_{1}, d_{2}, d_{3}$ (and similarly for $r=2$, but the program is trivial here). The very same linear programs (approximately) yield $F(n, r, 3)$ for $r \in\{2,3\}$ in the proof of the main result in [1], where the variables $d_{i}$ correspond to densities of overlapping triangle-free graphs.

Corollary 4.3. Let $r \in\{2,3\}$. For all $\varepsilon>0$, there exists $n_{0}>0$ such that the following holds. Let $n>n_{0}$ be an integer and let $A \subseteq[n]$ be such that $f(A, r) \geqslant f(n, r) \cdot 2^{-\varepsilon n /(200 r)}$. Then either $|A \triangle \mathrm{O}| \leqslant \varepsilon n$ or $\left|A \triangle I_{2}\right| \leqslant \varepsilon n$.

Proof. Choose $n_{0}>0$ sufficiently large so that the conclusion of Theorem 3.3 holds with parameter $\varepsilon /(200 r)$ and the conclusion of Lemma 4.1 holds with parameter $\varepsilon / 2$. Let $n \geqslant n_{0}$ be an integer and let $A \subseteq[n]$ be such that $f(A, r) \geqslant f(n, r) \cdot 2^{-\varepsilon n /(200 r)}$. From Theorem 3.3 obtain $A^{\prime} \subseteq A$ with $\left|A^{\prime}\right| \geqslant|A|-\varepsilon n /(200 r)$. Then

$$
g\left(A^{\prime}, r\right) \stackrel{(3.1)}{\geqslant} \frac{\log f(A, r)}{n}-\frac{\varepsilon}{200 r} \geqslant \frac{\log f(n, r)}{n}-\frac{\varepsilon}{100 r} \stackrel{(3.2)}{\geqslant} g(n, r)-\frac{\varepsilon}{100 r} .
$$

Lemma 4.1 implies that either $\left|A^{\prime} \triangle \mathrm{O}\right| \leqslant \varepsilon n / 2$ or $\left|A^{\prime} \triangle I_{2}\right| \leqslant \varepsilon n / 2$. The result follows. 
The first assertion $f(n, 3)=3^{n / 2+o(n)}$ of Theorem 1.3 follows immediately from Corollary 4.3.

\section{The proof of Theorem 1.2}

In this section we use Corollary 4.3 to prove Theorem 1.2. Before starting the proof, we need the following useful notion.

Definition 5.1. Given sets $S, B \subseteq[n]$, define the link graph of $B$ generated by $S$, denoted $L_{S}[B]$, as follows. We set $V\left(L_{S}[B]\right):=B$, and given $x, y \in B$, we have $x y \in E\left(L_{S}[B]\right)$ if and only if there is some $z \in S$ such that $\{x, y, z\}$ is a Schur triple, and $x, y, z$ are distinct. Note that $L_{S}[B]$ is a simple graph (i.e. it does not contain loops). If $S=\{v\}$, then we use the shorthand $L_{v}[B]:=L_{\{v\}}[B]$.

This notion is useful since each edge in a link graph represents some restriction of valid colourings, and so a large set of independent edges in a link graph limits the number of valid colourings.

Lemma 5.2. Let $n, r \in \mathbb{N}$ and let $A \subseteq[n]$. Let $x \in A$ and suppose that the link graph $L_{x}[A \backslash\{x\}]$ contains a matching $M_{x}$ of size $m$. Then

$$
f(A, r) \leqslant\left(r^{2}-1\right)^{m} \cdot r^{|A|-2 m} .
$$

Proof. We will bound $f(A, r)$ by re-constructing valid colourings using the following procedure, in which every choice yields a colouring $\tau: A \rightarrow[r]$ (which may or may not be valid).

(1) Let $\tau(x) \in[r]$ be arbitrary.

(2) For each edge $u v$ in $M_{x}$, choose $(\tau(u), \tau(v)) \in[r]^{2} \backslash\{(\tau(x), \tau(x))\}$.

(3) For each uncoloured $y \in A$, let $\tau(y) \in[r]$ be arbitrary.

To see that the procedure generates every valid $r$-colouring of $A$, we just need to check that every valid $r$-colouring $\sigma$ has the property in Step 2 (since all other choices were arbitrary). That is, for all $u v \in M_{x}, \sigma$ does not assign $u, v, x$ the same colour. But this is clear since $\{u, v, x\}$ is a Schur triple.

Therefore the number of colourings generated by the procedure is an upper bound for $f(A, r)$. Thus, using the fact that $\left|A \backslash\left(\{x\} \cup V\left(M_{x}\right)\right)\right|=|A|-2 m-1$, we have

$$
f(A, r) \leqslant r \cdot\left(r^{2}-1\right)^{m} \cdot r^{|A|-2 m-1}
$$

as required. 
We are now ready to prove Theorem 1.2.

Proof of Theorem 1.2. First recall that $2^{\lceil n / 2\rceil}$ is a lower bound for $f(n, 2)$ for all positive integers $n$, since we can always find a sum-free subset of $[n]$ of size $\lceil n / 2\rceil$, namely $\mathrm{O}$ or $I_{2}$, and colour it arbitrarily. Let $0<\varepsilon<1 / 200$ and apply Corollary 4.3 to obtain $n_{0}$. Now fix an integer $n>n_{0}$, and let $A \subseteq[n]$ be such that

$$
f(A, 2)=f(n, 2) \geqslant 2^{\lceil n / 2\rceil} .
$$

By Corollary 4.3, we have that either $|A \triangle \mathrm{O}| \leqslant \varepsilon n$; or $\left|A \triangle I_{2}\right| \leqslant \varepsilon n$. Thus $|A| \leqslant$ $\lceil n / 2\rceil+\varepsilon n$. We will use the following claim throughout the proof.

Claim 5.3. For all $x \in A$, every matching in $L_{x}[A \backslash\{x\}]$ has size less than $3 \varepsilon n$.

Indeed, if not, then there exists a matching of size exactly $3 \varepsilon n$. Lemma 5.2 implies that

$$
f(A, 2) \leqslant 3^{3 \varepsilon n} \cdot 2^{|A|-6 \varepsilon n} \leqslant 3^{3 \varepsilon n} \cdot 2^{\left\lceil\frac{n}{2}\right\rceil+\varepsilon n-6 \varepsilon n} \leqslant 2^{\left\lceil\frac{n}{2}\right\rceil-\frac{\varepsilon n}{5}},
$$

a contradiction.

To find large matchings in link graphs in the next two claims, we will use the fact that a graph $G$ with $e$ edges and maximum degree $\Delta$ contains a matching of size at least $e /(\Delta+1)$. This is an immediate consequence of Vizing's theorem on edge-colourings.

Case 1. $|A \triangle \mathrm{O}| \leqslant \varepsilon n$.

In this case, we will prove that $A=\mathrm{O}$. It suffices to show that $A \cap E=\emptyset$, since for any such set we have $f(A, 2)=2^{|A|}$, and thus $A=\mathrm{O}$ is clearly the unique extremal subset among such sets. Arguing by contradiction, the following claim together with Claim 5.3 will complete the proof of this case.

Claim 5.4. Suppose that there exists $x \in A \cap E$. Then $L_{x}[A \backslash\{x\}]$ contains a matching $M_{x}$ of size at least $9 \varepsilon n$.

Proof: Since $x$ is even, $A \cap \mathrm{O} \subseteq A \backslash\{x\}$, and thus $L_{x}[A \cap \mathrm{O}] \subseteq L_{x}[A \backslash\{x\}]$. Also, since $|A \triangle \mathrm{O}| \leqslant \varepsilon n$, it suffices to show that $L_{x}[\mathrm{O}]$ contains a matching of size at least $10 \varepsilon n$. First, we will assume that $x \leqslant n-60 \varepsilon n$. In $L_{x}[\mathrm{O}]$, every odd number $y$ has degree at least one, since either $x+y \leqslant n$, or $y-x \geqslant 1$ (or both). Moreover, $y$ has degree two if both hold, i.e. when $x+1 \leqslant y \leqslant n-x$, and there are at least $\lceil(n-2 x) / 2\rceil$ such $y$ (note that there could be vertices less than $x$ which have degree two). Therefore $e\left(L_{x}[\mathrm{O}]\right) \geqslant \frac{1}{2}(x+(n-2 x))$. By Vizing's theorem, $L_{x}[\mathrm{O}]$ contains a matching of size at least

$$
\frac{e\left(L_{x}[\mathrm{O}]\right)}{\Delta\left(L_{x}[\mathrm{O}]\right)+1} \geqslant \frac{n-x}{6} \geqslant 10 \varepsilon n
$$

as required. Now, if $x>n-60 \varepsilon n$, then $M=\{\{1, x-1\},\{3, x-3\}, \ldots,\{x / 2-1, x-$ $(x / 2-1)\}\} \subseteq L_{x}[\mathrm{O}]$ is a matching of size $\lfloor x / 4\rfloor \geqslant n / 4-20 \varepsilon n \geqslant 10 \varepsilon n$. 
Case 2. $\left|A \triangle I_{2}\right| \leqslant \varepsilon n$.

Now $A$ has few elements from $I_{1}$. We first show that the smallest element in $A$ cannot be far from $n / 2$. For all $i \in \mathbb{N}$, denote by $\delta_{i}$ the $i^{\text {th }}$ smallest element in $A$ (denoted so in analogy with the minimum degree of a graph). We may suppose that $\delta_{1} \in I_{1}$ since otherwise $A \subseteq I_{2}$ is sum-free and we are done.

Claim 5.5. $\delta_{1}>n / 2-12 \varepsilon n$.

Proof: Suppose not. Similar to Case 1, since $\delta_{1} \in I_{1}$, we have that $A \cap I_{2} \subseteq A \backslash\left\{\delta_{1}\right\}$. Therefore, it suffices to show that the link graph $L_{\delta_{1}}\left[A \cap I_{2}\right]$ contains a matching of size at least $3 \varepsilon n$ which contradicts Claim 5.3. Since $\left|A \triangle I_{2}\right| \leqslant \varepsilon n$, we only need to show that $L_{\delta_{1}}\left[I_{2}\right]$ contains a matching of size at least $4 \varepsilon n$. In $L_{\delta_{1}}\left[I_{2}\right]$, every element in $\left[\lfloor n / 2\rfloor+1,\lfloor n / 2\rfloor+\delta_{1}\right] \cup\left[n-\delta_{1}+1, n\right]$ has degree one, and all the other $\lceil n / 2\rceil-2 \delta_{1}$ elements have degree two. Therefore $e\left(L_{\delta_{1}}\left[I_{2}\right]\right) \geqslant \frac{1}{2}\left(2 \delta_{1}+2\left(\lceil n / 2\rceil-2 \delta_{1}\right)\right)=\lceil n / 2\rceil-\delta_{1}$. Therefore, by Vizing's theorem, $L_{\delta_{1}}\left[I_{2}\right]$ contains a matching of size at least

$$
\frac{e\left(L_{\delta_{1}}\left[I_{2}\right]\right)}{\Delta\left(L_{\delta_{1}}\left[I_{2}\right]\right)+1} \geqslant \frac{\left\lceil\frac{n}{2}\right\rceil-\delta_{1}}{3} \geqslant \frac{n}{6}-\frac{\delta_{1}}{3} \geqslant 4 \varepsilon n,
$$

a contradiction.

Define $k$ such that $A \cap I_{1}=\left\{\delta_{1}, \ldots, \delta_{k}\right\}$. Then $B:=2 \cdot\left(A \cap I_{1}\right)$ is a subset of $I_{2}$ by Claim 5.5. There are two ways of colouring each of the $k$ pairs $\left\{\delta_{i}, 2 \delta_{i}\right\}$, so

$$
f(A, 2) \leqslant 2^{k} \cdot 2^{\left|\left(A \cap I_{2}\right) \backslash B\right|} \leqslant 2^{k} \cdot 2^{\left|I_{2}\right|-k}=2^{\lceil n / 2\rceil}
$$

with equality in the second inequality if and only if $\left|\left(A \cap I_{2}\right) \backslash B\right|=\left|I_{2}\right|-k$, i.e. if and only if $I_{2} \backslash A \subseteq B$.

Claim 5.6. $\delta_{1}+\delta_{2} \geqslant n+1$.

Proof: Suppose that $\delta_{1}+\delta_{2} \leqslant n$. Then $\delta_{1} \in I_{1}$ (but $\delta_{2}$ may or may not be). Since $\overline{\delta_{1}+\delta_{2}} \notin B$, we have $\delta_{1}+\delta_{2} \in A$. Let $C:=\left\{\delta_{1}, \delta_{2}, 2 \delta_{1}, \delta_{1}+\delta_{2}, 2 \delta_{2}\right\}$. Then $\left\{\delta_{1}, \delta_{2}, \delta_{1}+\delta_{2}\right\} \subseteq$ $A \cap C \subseteq C$, and one can easily check that there are at most $6=2^{4}-2$ valid colourings of $A \cap C$, the missing colourings being the two monochromatic ones.

Suppose first that $\delta_{2} \in I_{1}$. Then $I_{2} \cap C=\left\{2 \delta_{1}, \delta_{1}+\delta_{2}, 2 \delta_{2}\right\}$. So

$$
f(A, 2) \leqslant 2^{k-2} \cdot 2^{\left|I_{2} \backslash C\right|-(k-2)} \cdot 6=6 \cdot 2^{\left|I_{2}\right|-3}=\frac{3}{4} \cdot 2^{\lceil n / 2\rceil},
$$

a contradiction. Suppose instead that $\delta_{2} \in I_{2}$. Then $k=1$ and $I_{2} \cap C=\left\{\delta_{2}, 2 \delta_{1}, \delta_{1}+\delta_{2}\right\}$, and

$$
f(A, 2) \leqslant 2^{\left|I_{2} \backslash C\right|} \cdot 6=6 \cdot 2^{\left|I_{2}\right|-3}=\frac{3}{4} \cdot 2^{\lceil n / 2\rceil} .
$$

Thus $\delta_{1}+\delta_{2} \geqslant n+1$, as required.

So certainly $\left|A \cap I_{1}\right| \leqslant 1$, and $I_{2} \backslash A \subseteq\left\{2 \delta_{1}\right\}$, but we can say more. If $A \cap I_{1}=\emptyset$ then $A=I_{2}$ and we are done. So we may assume that $A \cap I_{1}=\left\{\delta_{1}\right\}$. Suppose $\delta_{2} \neq\lfloor n / 2\rfloor+1$. 
Then $\lfloor n / 2\rfloor+1$ is missing from $A$ and hence $2 \delta_{1}=\lfloor n / 2\rfloor+1$, a contradiction to Claim 5.5. Thus $\delta_{2}=\lfloor n / 2\rfloor+1$ and therefore

$$
n+1 \leqslant \delta_{1}+\delta_{2} \leqslant 2\lfloor n / 2\rfloor+1 .
$$

So $n$ is even and we have equality if and only if $\left(\delta_{1}, \delta_{2}\right)=(n / 2, n / 2+1)$. Since $I_{2} \backslash A \subseteq\{n\}$, there are two candidates for extremal sets: $[n / 2, n-1]$ and $[n / 2, n]$. It is easy to see that both have $2^{n / 2}$ valid 2-colourings. This completes the proof of Theorem 1.2.

\section{The proof of Theorem 1.4}

To prove Theorem 1.4, by Theorem 3.3, it suffices to determine $g(n, r)$ asymptotically. Our aim is to show that

$$
g(n, 4)=1+o(1) \quad \text { and } \quad g(n, 5)=\frac{1}{4} \log 30+o(1) .
$$

Recall from the proof of Lemma 4.1 that we reduced the problem of asymptotically determining $g(n, 3)$ to solving a linear program. Indeed, we let $\left(A_{1}, A_{2}, A_{3}\right)$ be a tuple of sum-free subsets with intersection vector $\left(d_{1}, d_{2}, d_{3}\right)$, and found a reduction of Problem 2 into a linear program in variables $d_{1}, d_{2}, d_{3}$. Our task was then to maximise $\sum_{i \in[3]} d_{i} \log i$ subject to $d_{1}, d_{2}, d_{3} \geqslant 0$ and $\sum_{i \in[3]} i d_{i} \leqslant \frac{3}{n}\left\lceil\frac{n}{2}\right\rceil$.

So to prove Theorem 1.4, we will again reduce Problem 2 to a linear program in variables $d_{1}, \ldots, d_{r}$ and an additional slack variable $a$ (defined below) for $r \in\{4,5\}$.

Given $n \in \mathbb{N}$, let $A_{1}, \ldots, A_{r} \in[n]$ be maximal sum-free sets. Throughout the rest of this section, define $D_{1}, \ldots, D_{r}, d_{1}, \ldots, d_{r}$ as in Problem 2. So $D_{i}$ is the set of all elements that are in exactly $i$ different $A_{j}$ 's, and $d_{i}:=\left|D_{i}\right| / n$. Further, we let $C$ be the subset of $[r]$ such that $A_{i}$ is a type (a) set for every $i \in C$, and a type (b) or (c) set, for every $i \in[r] \backslash C$. Recall that a type (a) set $S$ has $|S| \leqslant 2 n / 5+1$, a type (b) set consists of odd numbers, and a type (c) set $S$ has $|S| \leqslant \min (S)$. We define

$$
a:=\frac{1}{n} \sum_{i \in[r] \backslash C}\left(\left\lceil\frac{n}{2}\right\rceil-\left|A_{i}\right|\right)=\frac{(r-|C|)}{n} \cdot\left\lceil\frac{n}{2}\right\rceil-\frac{1}{n} \sum_{i \in[r] \backslash C}\left|A_{i}\right| .
$$

An important observation is that, if $A$ is a maximal sum-free set of type (b), then $A$ is precisely $\mathrm{O}$, the set of odd integers in $[n]$. Thus

$$
a=\frac{1}{n} \sum_{i: A_{i} \text { of type }(c)}\left(\left\lceil\frac{n}{2}\right\rceil-\left|A_{i}\right|\right) .
$$

Definition 6.1. Let $r \in \mathbb{N}$ and $\varepsilon>0$. Let $0 \leqslant f_{1}, \ldots, f_{r} \leqslant 1$ be variables. Suppose that

$$
\alpha_{i 1} f_{1}+\ldots+\alpha_{i r} f_{r}+\alpha_{i} a \leqslant \beta_{i} \text { for all } i \leqslant N(r)
$$


where $N(r)$ is a positive integer depending only on $r$; and $\alpha_{i j}, \beta_{i}, \alpha_{i} \in \mathbb{R}$ for all $i \leqslant N(r)$ and $j \leqslant r$. We say that this set $\mathcal{C}$ of inequalities is $(\varepsilon, r)$-sufficient if

$$
\max \sum_{i \in[r]} f_{i} \log i \quad \text { subject to } \quad \mathcal{C} \quad \text { is at most } \max \left\{\frac{1}{2} \log r, \frac{1}{4} \log \left(r\left\lfloor r^{2} / 4\right\rfloor\right)\right\}+\varepsilon .
$$

When $\left(d_{1}, \ldots, d_{r}\right)$ playing the roles of $\left(f_{1}, \ldots, f_{r}\right)$ satisfies $\mathcal{C}$, we say that $\mathcal{C}$ is a family of constraints for $A_{1}, \ldots, A_{r}$.

We will always use $d_{i}$ instead of $f_{i}$ since we are only interested in $\mathcal{C}$ which are a family of constraints for $A_{1}, \ldots, A_{r}$. However, we would like these constraints to be independent of $n$, which of course $A_{1}, \ldots, A_{r}$ are not. As an example, when $r=3$, we showed that, for every $\varepsilon>0$, sufficiently large $n \in \mathbb{N}$ and sum-free subsets $A_{1}, A_{2}, A_{3}$ of $[n]$, the family

$$
\left\{d_{1} \geqslant 0 ; d_{2} \geqslant 0 ; d_{3} \geqslant 0 ; d_{1}+2 d_{2}+3 d_{3} \leqslant \frac{3}{2}+\frac{\varepsilon}{300}\right\}
$$

of constraints is $(\varepsilon, 3)$-sufficient. Indeed, the final constraint we actually derived was $d_{1}+2 d_{2}+3 d_{3} \leqslant 3 / n \cdot\lceil n / 2\rceil$, but we can replace the right-hand side by the weaker $3 / 2+\varepsilon / 300$ to obtain a family which is still a family of constraints for $A_{1}, A_{2}, A_{3}$, but is independent of $n$.

The proof of Theorem 1.4 follows from the next lemma.

Lemma 6.2. Let $r \in\{4,5\}$. For all $\varepsilon>0$, there exists an $n_{0}>0$ such that for all integers $n \geqslant n_{0}$, every choice of maximal sum-free subsets $A_{1}, \ldots, A_{r}$ of $[n]$ has a family of $(\varepsilon, r)$-sufficient constraints.

Indeed, suppose that the lemma holds. The construction after the statement of Theorem 1.4 shows that, whenever $n$ is a sufficiently large integer, we have $f(n, 4) \geqslant 16^{\lfloor n / 4\rfloor}>$ $2^{(1-\varepsilon) n}$. Thus it suffices to find $n_{0}>0$ such that $f(n, 4) \leqslant 2^{(1+\varepsilon) n}$ whenever $n \geqslant n_{0}$ is an integer. Choose $n_{0}$ so that the conclusions of Theorem 3.3 (with $r=4$ ) and Lemma 6.2 hold for parameter $\varepsilon / 2$ and all $n \geqslant n_{0}$. Now let $n \geqslant n_{0}$ be an arbitrary integer. By Remark 3.2, there are maximal sum-free subsets $A_{1}, \ldots, A_{4}$ of $[n]$ such that $g\left(A_{1}, \ldots, A_{4}\right)=g(n, 4)$. By Lemma $6.2, A_{1}, \ldots, A_{4}$ has a family of $(\varepsilon / 2,4)$-sufficient constraints. Thus $g(n, 4)=g\left(A_{1}, \ldots, A_{4}\right)=\sum_{i \in[4]} d_{i} \log i \leqslant 1+\varepsilon / 2$. Then

$$
f(n, 4) \stackrel{(3.2)}{\leqslant} 2^{(g(n, 4)+\varepsilon / 2) n} \leqslant 2^{(1+\varepsilon) n},
$$

as required. The case $r=5$ is almost identical.

For each $r=4,5$, we split the proof of Lemma 6.2 into cases depending on the structure of $A_{1}, \ldots, A_{r}$ (obtained from Theorem 2.1). Then, in each case, we find a family of constraints which is $(\varepsilon, r)$-sufficient. Given a family $\mathcal{C}$ of inequalities, we must

1. show that it is a family of constraints for $A_{1}, \ldots, A_{r}$, i.e. that each inequality holds; then 
2. show that it is $(\varepsilon, r)$-sufficient, i.e. consider the linear program $\max \sum_{i \in[r]} d_{i} \log i$ subject to $\mathcal{C}$, and show that its optimal solution is at most the required value.

Since it is only a series of tedious calculations, we defer the details of (2) to the appendix, and limit ourselves to some remarks here.

\subsection{Achieving (2): Solving linear programs}

Since there are many cases (depending on the structure of $A_{1}, \ldots, A_{r}$ ), and sometimes rather a lot of inequalities in each family of constraints $\mathcal{C}$, where possible, we use Mathematica to solve the resulting linear program $\max _{i \in[r]} d_{i} \log i$ subject to $\mathcal{C}$. Suppose that $r=4$ (when $r=5$ the situation is similar). Given $A_{1}, \ldots, A_{4}$, an $(\varepsilon, 4$ )-sufficient family $\mathcal{C}$ is such that $\sum_{i \in[4]} d_{i} \log i \leqslant 1+\varepsilon$.

There are two cases, depending on whether the 4-tuple $\left(A_{1}, A_{2}, A_{3}, A_{4}\right)$ is close to extremal or not, namely if $g\left(A_{1}, A_{2}, A_{3}, A_{4}\right)$ is close to the maximum possible value or not. Suppose that there is some specific value of $\varepsilon$, say $\varepsilon=1 / 1000$, and a family $\mathcal{C}$ of constraints which is $(1 / 1000,4)$-sufficient, for which Mathematica shows the output $\sum_{i \in[4]} d_{i} \log i \leqslant 0.999$. The level of accuracy of the program is enough for us to know that certainly $g\left(A_{1}, \ldots, A_{4}\right)=\sum_{i \in[4]} d_{i} \log i<1-1 / 2000$. So in this case, we are done, and in fact since this number is less than our lower bound (3.6) by some absolute constant, we see that $A_{1}, \ldots, A_{4}$ cannot be close to extremal.

If instead, given input $\varepsilon=1 / 1000$, Mathematica shows an output

$$
0.999<\sum_{i \in[4]} d_{i} \log i \leqslant 1.001
$$

say, we need to be more careful. In this case, we will write out the dual program of $\max \sum_{i \in[4]} d_{i} \log i$ subject to $\mathcal{C}$, which is a minimisation problem. We then exhibit a feasible solution to the dual which is at most $1+\varepsilon$. By the weak duality theorem, we see that $\max \sum_{i \in[4]} d_{i} \log i \leqslant 1+\varepsilon$, as required.

\subsection{Linear constraints for general $r$}

To achieve (1), we will first derive a set of linear constraints which apply for any number $r$ of colours.

Lemma 6.3. For all $\varepsilon>0$ and integers $r \geqslant 4$, there exists an $n_{0}>0$ such that the following holds. Let $n, s, t \in \mathbb{N}$ be such that $n \geqslant n_{0}$ and $s+t \leqslant r$. Also, let $A_{1}, \ldots, A_{r}$ be maximal sum-free subsets of $[n]$ such that $s$ of them are of type (b), $t$ of them are of type (c), and $\left(d_{1}, \ldots, d_{r}\right)$ is their intersection vector. Then

$$
\sum_{i \in[r]} i d_{i} \leqslant \frac{r}{2}-\frac{r-s-t}{10}-a+\varepsilon \quad \text { and } \quad d_{r-1}+d_{r} \leqslant \frac{1}{2}+\varepsilon .
$$


Proof. Let $n_{0}:=r / \varepsilon$ and let $n \geqslant n_{0}$ be an integer. For the first inequality, by (6.1) and the definition of type (a) sets, we have

$$
\sum_{i \in[r]} i d_{i} \leqslant \frac{s+t}{n} \cdot\left\lceil\frac{n}{2}\right\rceil+\left(\frac{2}{5}+\frac{1}{n}\right)(r-s-t)-a \leqslant \frac{r}{2}-\frac{r-s-t}{10}-a+\varepsilon,
$$

where the last inequality follows from $n \geqslant r / \varepsilon$. To prove the second part of the lemma, since $\varepsilon \geqslant r / n \geqslant 1 / n$, it suffices to show that the set $D_{r} \cup D_{r-1}$ is sum-free. Assume to the contrary that there exist $x_{1}, x_{2}, x_{3} \in D_{r} \cup D_{r-1}$ such that $x_{1}+x_{2}=x_{3}$. For every $i \in[3]$, define $I_{i}:=\left\{j: j \in[r]\right.$ and $\left.x_{i} \in A_{j}\right\}$ and $\overline{I_{i}}=[r] \backslash I_{i}$. Since $x_{i} \in D_{r} \cup D_{r-1}$, we have $\left|\overline{I_{i}}\right| \leqslant 1$ for every $i \in[3]$, and therefore $\left|\bigcap_{i \in[3]} I_{i}\right| \geqslant r-\sum_{i \in[3]}\left|\overline{I_{i}}\right| \geqslant r-3 \geqslant 1$, where the last inequality follows from $r \geqslant 4$. Therefore, there exists an $i \in[r]$ such that $x_{1}, x_{2}, x_{3} \in A_{i}$, which contradicts $A_{i}$ being sum-free.

We will use the next two simple facts repeatedly. Recall that $d(A)=|A| / n$ is the density of a set $A$.

Observation 6.4. Let $k \in[r]$ and suppose that $A_{1}, \ldots, A_{k}$ are of type (c). Then

(i) $\min \left(A_{i}\right) \geqslant\lceil n / 2\rceil-$ an for all $i \in[k]$.

(ii) $\sum_{i \in[k]} d\left(A_{i}\right) \geqslant k \cdot \frac{\lceil n / 2\rceil}{n}-a$.

Proof. The second equality for parameter a just after equation (6.1) states that an is the sum of $\lceil n / 2\rceil-\left|A_{i}\right|$ over $i \in[k]$. Thus, for (i), using the definition of type (c) sets, every $i \in[k]$ satisfies $\min \left(A_{i}\right) \geqslant\left|A_{i}\right| \geqslant\lceil n / 2\rceil-a n$, and for (ii), $\left|A_{1}\right|+\ldots+\left|A_{k}\right|=$ $k\lceil n / 2\rceil-a n$.

The next lemma concerns the size of the intersection of type (c) sets.

Lemma 6.5. For all $\varepsilon>0$ and positive integers $r$, there exists an $n_{0}>0$, such that the following holds. For every integer $n \geqslant n_{0}$, let $A_{1}, \ldots, A_{r}$ be maximal sum-free subsets of $[n]$ such that there is some $k \leqslant r$ for which $A_{1}, \ldots, A_{k} \subseteq[n]$ are type (c) sets. Then

$$
d\left(\cap_{i \in[k]} A_{i}\right) \geqslant \frac{1}{2}-k a-\varepsilon .
$$

Proof. Let $n_{0}:=k / \varepsilon$ and let $n>n_{0}$ be an integer and $A_{1}, \ldots, A_{r}$ be subsets of $[n]$ as in the statement. Let $A^{*}=\cap_{i \in[k]} A_{i}$. It suffices to show that $\left|A^{*}\right| \geqslant\lceil n / 2\rceil-(k-1)-k a n$. By Observation 6.4(i), for all $i \in[k], \min \left(A_{i}\right) \geqslant\lceil n / 2\rceil-$ an, and therefore $A_{i} \subseteq I_{a}:=$ $[\lceil n / 2\rceil-a n, n]$. Every integer $x$ in the subset $L:=I_{a} \backslash A^{*}$ lies in at most $k-1$ of the $A_{i}$ 's. Assume to the contrary that $\left|A^{*}\right|<\lceil n / 2\rceil-(k-1)-k a n$. Then

$$
\begin{aligned}
\sum_{i \in[k]}\left|A_{i}\right| & \leqslant k \cdot\left|A^{*}\right|+(k-1)|L|=(k-1)\left|I_{a}\right|+\left|A^{*}\right| \\
& <(k-1)(n-(\lceil n / 2\rceil-a n)+1)+\lceil n / 2\rceil-(k-1)-k a n \\
& =(k-1) n-(k-2)\lceil n / 2\rceil-\text { an } .
\end{aligned}
$$


But Observation 6.4(ii) implies that $\sum_{i \in[k]}\left|A_{i}\right| \geqslant k\lceil n / 2\rceil-$ an. Together with (6.2), we get $2(k-1)\lceil n / 2\rceil<(k-1) n$, a contradiction.

Throughout the rest of the paper, given $a$ defined by (6.1), we will let

$$
J_{1}:=\left[1,\left\lfloor\frac{n}{2}\right\rfloor-a n\right], \quad J_{2}:=\left[\left\lfloor\frac{n}{2}\right\rfloor-a n+1, n\right], \quad \text { and } \quad J_{3}:=\left[\left\lfloor\frac{n}{2}\right\rfloor-a n+1,\left\lfloor\frac{n}{2}\right\rfloor\right]
$$

and will refer to $J_{1}, J_{2}$, and $J_{3}$ as the first, second, and middle interval respectively. Note that by definition an is an integer, and the set $J_{2} \backslash J_{3}=I_{2}$ is a sum-free set of maximum size $\lceil n / 2\rceil$. The following observation is a straightforward consequence of Observation 6.4(i) and the fact that the unique maximal sum-free subset of type (b) is $\mathrm{O}$.

Observation 6.6. (i) If $A_{i}$ is a maximal sum-free set of type (c), then $\left|A_{i} \backslash J_{2}\right| \leqslant 1$.

(ii) For all $\varepsilon>0$ and positive integers $r$, there exists $n_{0}>0$ such that the following holds. Let $A_{1}, \ldots, A_{r}$ be maximal sum-free subsets of $[n]$ such that $s$ of them are of type (b), $t$ of them are of type (c), and $r-s-t$ of them are of type (a). Then

$$
d\left(\left(D_{0} \cup \ldots \cup D_{r-s-t}\right) \cap J_{1} \cap E\right), d\left(\left(D_{s} \cup \ldots \cup D_{r-t}\right) \cap J_{1} \cap O\right) \geqslant 1 / 4-a / 2-\varepsilon .
$$

The final result in this subsection states some constraints involving the intervals $J_{2}$ and $J_{3}$.

Lemma 6.7. For all $\varepsilon>0$ and positive integers $r$, there exists $n_{0}>0$ such that the following holds. Let $k \leqslant r$ and $n \geqslant n_{0}$ be positive integers. Let $A_{1}, \ldots, A_{k} \subseteq[n]$ be type (c) maximal sum-free sets and define $q_{i}:=d\left(A_{i} \cap J_{3}\right)$ for all $i \in[k]$. Then

$$
\sum_{i \in[k]} q_{i} \leqslant a+\varepsilon \quad \text { and } \quad d\left(\left(\cap_{i \in[k]} A_{i}\right) \cap I_{2}\right) \geqslant \frac{1}{2}-\sum_{i \in[k]} q_{i}-a-\varepsilon \geqslant \frac{1}{2}-2 a-2 \varepsilon .
$$

Proof. Let $n_{0}:=r / \varepsilon$ and let $n \geqslant n_{0}$ be an integer. Since $A_{i}$ has $q_{i} n$ elements in $J_{3}$, we have that $\min \left(A_{i}\right) \leqslant\left\lfloor\frac{n}{2}\right\rfloor+1-q_{i} n$ for all $i \in[k]$. Using Observation 6.4(ii) and the definition of type (c) sets, we have

$$
\frac{k}{n}\left\lceil\frac{n}{2}\right\rceil-a \leqslant \sum_{i \in[k]} d\left(A_{i}\right)=\sum_{i \in[k]} \frac{\left|A_{i}\right|}{n} \leqslant \sum_{i \in[k]} \frac{\min \left(A_{i}\right)}{n} \leqslant \frac{k}{n}\left(\left\lceil\frac{n}{2}\right\rceil+1\right)-\sum_{i \in[k]} q_{i} .
$$

Thus $\sum_{i \in[k]} q_{i} \leqslant k / n+a \leqslant a+\varepsilon$, proving the first inequality. To prove (ii), let $B:=$ $I_{2} \backslash \cap_{i \in[k]} A_{i}$. In other words, $B$ is the set of all elements in $I_{2}=J_{2} \backslash J_{3}$ that are missing from at least one of the $A_{1}, \ldots, A_{k}$. By Observation 6.6(i),

$$
\sum_{i \in[k]} d\left(A_{i}\right) \leqslant \sum_{i \in[k]} q_{i}+\sum_{i \in[k]} d\left(A_{i} \cap\left(J_{2} \backslash J_{3}\right)\right)+\frac{k}{n} \leqslant \sum_{i \in[k]} q_{i}+\frac{k}{n}\lceil n / 2\rceil-d(B)+\varepsilon .
$$

Thus

$$
d(B) \leqslant \sum_{i \in[k]} q_{i}+\frac{k}{n}\lceil n / 2\rceil-\sum_{i \in[k]} d\left(A_{i}\right)+\varepsilon \stackrel{(6.4)}{\leqslant} \sum_{i \in[k]} q_{i}+a+\varepsilon,
$$

and the second required inequality follows. 


\subsection{The 4 colour case}

Proof of Lemma 6.2 when $r=4$. Let $\varepsilon>0$ and choose $\varepsilon^{\prime}, n_{0}>0$ such that $1 / n_{0} \ll$ $\varepsilon^{\prime} \ll \varepsilon \leqslant 1 / 100$. (we may assume the last inequality without loss of generality). Let $n \geqslant n_{0}$ be an integer and let $A_{1}, \ldots, A_{4} \subseteq[n]$ be maximal sum-free sets with intersection vector $\left(d_{1}, \ldots, d_{4}\right)$ as defined in Problem 2 . We need to obtain a family of $(\varepsilon, 4)$-sufficient constraints. We have the following set of basic constraints which will be used throughout the proof:

$$
\left\{\begin{array}{l}
d_{i} \geqslant 0 \text { for all } i \in\{0, \ldots, 4\} \\
\sum_{i \in\{0, \ldots, 4\}} d_{i} \leqslant 1, \\
d_{3}+d_{4} \leqslant\left\lceil\frac{n}{2}\right\rceil / n \leqslant \frac{1}{2}+\varepsilon^{\prime},
\end{array}\right.
$$

where the last inequality follows from Lemma 6.3.

Suppose first that there is an $i \in[4]$ for which $A_{i}$ is of type (a). By Lemma 6.3, we have that

$$
\sum_{i \in[4]} i d_{i} \leqslant 19 / 10+\varepsilon^{\prime}
$$

(The ${ }^{*}$ denotes the fact that this inequality does not hold in Case 1 onwards.) The family $\left\{\left(\mathrm{C} 0^{*}\right),(\mathrm{C} 1)\right\}$ is $(\varepsilon, 4)$-sufficient (for justification here and from now on, see the appendix).

Thus we can assume that all of $A_{1}, \ldots, A_{4}$ are of type (b) or (c). Let $s$ be the number of $A_{i}$ of type (b) and $t$ the number of $A_{i}$ of type (c) (so $s+t=4$ ). Define $a$ as in (6.1). By Lemma 6.3 (with $\varepsilon^{\prime}$ playing the role of $\varepsilon$ ), we have

$$
\sum_{i \in[4]} i d_{i} \leqslant 2-a+\varepsilon^{\prime}
$$

Suppose that $a \geqslant 1 / 10$. Then (C2) implies that $\left(\mathrm{C} 0^{*}\right)$ holds. But, as we have seen, $\left\{\left(\mathrm{C} 0^{*}\right),(\mathrm{C} 1)\right\}$ is an $(\varepsilon, 4)$-sufficient family. Hence, throughout the rest of the proof, we can assume that

$$
a<1 / 10
$$

Given $a$, define $J_{1}, J_{2}, J_{3}$ as in (6.3). By Observation 6.6(ii),

$$
\left.\begin{array}{l}
d_{0} \geqslant d\left(D_{0} \cap J_{1}\right) \\
d_{s} \geqslant d\left(D_{s} \cap J_{1}\right)
\end{array}\right\} \geqslant \frac{1}{4}-\frac{a}{2}-\varepsilon^{\prime}
$$

Case 1. $s \in\{0,1\}$.

Suppose first that $s=0$. Then $A_{1}, \ldots, A_{4}$ are all type (c), so Lemma 6.5 (with $\varepsilon^{\prime}$ playing the role of $\varepsilon$ ) implies that

$$
d_{4} \geqslant \frac{1}{2}-4 a-\varepsilon^{\prime}
$$


The family $\{(\mathrm{C} 1),(\mathrm{C} 2),(\mathrm{C} 4),(\mathrm{C} 5)\}$ is $(\varepsilon, 4)$-sufficient. (We could also include (C3) but it is not needed.)

Suppose instead that $s=1$. Without loss of generality, let $A_{4}$ be the only type (b) set. By Lemma 6.5 (with $\varepsilon^{\prime}$ playing the role of $\varepsilon$ ), $d\left(\cap_{i \in[3]} A_{i}\right) \geqslant 1 / 2-3 a-\varepsilon^{\prime}$. By Observation 6.6(i), $\left|\cap_{i \in[3]} A_{i} \backslash J_{2}\right| \leqslant 1$. Also, every element $x \in \cap_{i \in[3]} A_{i}$ is in $D_{3}$ if it is even, and $D_{4}$ if it is odd. Thus

$$
\begin{aligned}
d_{3} & \geqslant d\left(\left(\cap_{i \in[3]} A_{i}\right) \cap J_{2}\right)-d\left(O \cap J_{2}\right) \geqslant d\left(\cap_{i \in[3]} A_{i}\right)-\frac{1}{n}-\frac{1}{n} \cdot\left\lceil\left(n-\left\lfloor\frac{n}{2}\right\rfloor+a n\right) / 2\right\rceil \\
& \geqslant \frac{1}{2}-3 a-\varepsilon^{\prime}-\frac{1}{n}-\frac{1}{4}-\frac{a}{2}-\frac{1}{2 n} \geqslant \frac{1}{4}-\frac{7 a}{2}-2 \varepsilon^{\prime} .
\end{aligned}
$$

The family $\{(\mathrm{C} 1),(\mathrm{C} 2),(\mathrm{C} 3),(\mathrm{C} 4),(\mathrm{C} 6)\}$ is $(\varepsilon, 4)$-sufficient. This completes the proof of Case 1.

Case 2. $t \in\{0,1\}$.

Suppose first that $t=0$. Then all of $A_{1}, \ldots, A_{4}$ are of type (b), so $D_{0}$ is the set of evens, $D_{4}$ is the set of odds, and all the other $D_{i}$ 's are empty. Therefore

$$
g\left(A_{1}, \ldots, A_{4}\right)=2 \cdot d_{4}=\frac{2}{n} \cdot\left\lceil\frac{n}{2}\right\rceil \leqslant 1+\varepsilon^{\prime},
$$

as required. Suppose instead that $t=1$. Then $D_{0} \cup D_{1}$ contains every even integer, and therefore

$$
d_{0}+d_{1} \geqslant \frac{1}{n} \cdot\left\lfloor\frac{n}{2}\right\rfloor \geqslant \frac{1}{2}-\varepsilon^{\prime} .
$$

The family $\{(\mathrm{C} 1),(\mathrm{C} 2),(\mathrm{C} 3),(\mathrm{C} 4),(\mathrm{C} 7)\}$ is $(\varepsilon, 4)$-sufficient. This completes the proof of Case 2. Therefore, the only remaining case is the following.

Case 3. $s=t=2$.

We will prove that the following constraints hold.

$$
\begin{aligned}
& d_{2} \geqslant \frac{1}{2}-\frac{5 a}{2}-2 \varepsilon^{\prime}, \\
& d_{3} \leqslant 3 a+\varepsilon^{\prime} .
\end{aligned}
$$

Without loss of generality, we can assume that $A_{1}$ and $A_{2}$ are of type (c). We first prove (C8). By (C4), we have that $d\left(D_{2} \cap J_{1}\right) \geqslant 1 / 4-a / 2-\varepsilon^{\prime}$. Therefore, we only need to show that $d\left(D_{2} \cap J_{2}\right) \geqslant 1 / 4-2 a-\varepsilon^{\prime}$. Let $B:=A_{1} \cap A_{2} \cap\left(J_{2} \backslash J_{3}\right)$. Then $B \cap E \subseteq D_{2} \cap J_{2}$. Lemma 6.7 applied with parameter $\varepsilon^{\prime} / 4$ implies that $d(B) \geqslant 1 / 2-2 a-\varepsilon^{\prime} / 2$. Further, $J_{2} \backslash J_{3}$ is an interval of length $\lceil n / 2\rceil$ so contains at most $n / 4+1$ odd elements. Thus

$$
d\left(D_{2} \cap J_{2}\right) \geqslant d(B \cap E)=d(B)-d(B \cap O) \geqslant \frac{1}{2}-2 a-\frac{\varepsilon^{\prime}}{2}-d\left(\left(J_{2} \backslash J_{3}\right) \cap O\right) \geqslant \frac{1}{4}-2 a-\varepsilon^{\prime} .
$$


We now prove (C9). By Observation 6.6(i), we have $\left|A_{1} \cap J_{1}\right|,\left|A_{2} \cap J_{1}\right| \leqslant 1$. Thus $\left|D_{3} \cap J_{1}\right| \leqslant 2$. Further, we have $A_{1} \cap A_{2} \cap E \subseteq D_{2}$ and $A_{1} \cap A_{2} \cap O \subseteq D_{4}$. In particular, $\left(A_{1} \cap A_{2}\right) \cap D_{3}=\emptyset$. Therefore

$d_{3}=d\left(D_{3} \cap J_{1}\right)+d\left(D_{3} \cap J_{2}\right) \leqslant \frac{2}{n}+d\left(J_{2}\right)-d(B) \stackrel{(6.3)}{\leqslant} \frac{2}{n}+1-\frac{1}{n} \cdot\lfloor n / 2\rfloor+a-\frac{1}{2}+2 a+\frac{\varepsilon^{\prime}}{2} \leqslant 3 a+\varepsilon^{\prime}$,

as required. But $\{(\mathrm{C} 1),(\mathrm{C} 2),(\mathrm{C} 3),(\mathrm{C} 8),(\mathrm{C} 9)\}$ is an $(\varepsilon, 4)$-sufficient family. This completes the proof of Case 3 , the final case.

\subsection{The 5 colour case}

Proof of Lemma 6.2 in the case $r=5$. Let $\varepsilon>0$ and choose $\varepsilon^{\prime}, n_{0}>0$ such that $1 / n_{0} \ll$ $\varepsilon^{\prime} \ll \varepsilon \leqslant 1 / 200$. (we may assume the last inequality without loss of generality). Let $n>n_{0}$ be an integer and let $A_{1}, \ldots, A_{5} \subseteq[n]$ be maximal sum-free sets with intersection vector $\left(d_{1}, \ldots, d_{5}\right)$. We have the following basic constraints which will be used throughout the proof.

$$
\left\{\begin{array}{l}
d_{i} \geqslant 0 \text { for all } i \in\{0, \ldots, 5\} \\
\sum_{i \in\{0, \ldots, 5\}} d_{i} \leqslant 1
\end{array}\right.
$$

Suppose first that $A_{1}, A_{2}$ are of type (a). Now Lemma 6.3 (with $\varepsilon^{\prime}$ playing the role of $\varepsilon$ ) implies that

$$
\sum_{i \in[5]} i d_{i} \leqslant 23 / 10+\varepsilon^{\prime}
$$

(The ${ }^{*}$ denotes the fact that this inequality does not hold in Case 1 onwards.) But $\left\{\left(\mathrm{D} 0^{*}\right),(\mathrm{D} 1)\right\}$ is an $(\varepsilon, 5)$-sufficient family.

Let $s$ be the number of $A_{i}$ of type (b) and $t$ the number of $A_{i}$ of type (c). So we may assume that $s+t \in\{4,5\}$.

Define $a$ as in (6.1). Lemma 6.3 implies that

$$
\sum_{i \in[5]} i d_{i} \leqslant \begin{cases}5 / 2-a+\varepsilon^{\prime}, & \text { if } s+t=5 \\ 12 / 5-a+\varepsilon^{\prime}, & \text { if } s+t=4 .\end{cases}
$$

Suppose now that $s+t=5$ and $a \geqslant 1 / 5$; or $s+t=4$ and $a \geqslant 1 / 10$. Then (D2) implies that $\left(\mathrm{D}^{*}\right)$ holds. But, as above, $\left\{\left(\mathrm{D} 0^{*}\right),(\mathrm{D} 1)\right\}$ is an $(\varepsilon, 5)$-sufficient family. Thus we may assume that

$$
\text { if } s+t=5 \text {, then } a<1 / 5 ; \quad \text { and if } s+t=4 \text {, then } a<1 / 10 \text {. }
$$

By Observation 6.6(ii), we have

$$
\left.\begin{array}{r}
d_{0} \geqslant d\left(D_{0} \cap J_{1} \cap E\right) \\
d_{s} \geqslant d\left(D_{s} \cap J_{1} \cap \mathrm{O}\right)
\end{array}\right\} \geqslant \frac{1}{4}-\frac{a}{2}-\varepsilon^{\prime}, \quad \text { if } s+t=5,
$$


We work through some cases depending on the values of $(s, t)$, in increasing order of complexity.

Case $1.5-s \leqslant 1$ or $5-t \leqslant 1$.

In this case, we will see that

$$
d_{0}+d_{1} \geqslant 1 / 2-a-\varepsilon^{\prime}
$$

Suppose first that $5-s \leqslant 1$. Now at most one $A_{i}$ can contain an even number, so $d_{0}+d_{1} \geqslant|E| / n \geqslant 1 / 2-\varepsilon^{\prime} \geqslant 1 / 2-a-\varepsilon^{\prime}$, as required. Suppose secondly that $5-t \leqslant 1$. Then summing the inequalities in each of (6.6) and (6.7) implies that $d_{0}+d_{1} \geqslant 1 / 2-a-\varepsilon^{\prime}$. But $\{(\mathrm{D} 1),(\mathrm{D} 2),(\mathrm{D} 3),(\mathrm{D} 4)\}$ is an $(\varepsilon, 5)$-sufficient family.

The remaining cases are $(s, t) \in\{(1,3),(3,1),(2,3),(3,2),(2,2)\}$.

Case 2. $(s, t) \in\{(1,3),(3,1)\}$.

Suppose that $A_{5}$ is the only set of type (a). By (6.7), we have that

$$
d_{0}+d_{1} \geqslant 1 / 4-a / 2-\varepsilon^{\prime} .
$$

Suppose first that $(s, t)=(1,3)$. Then summing the inequalities in (6.7) implies that

$$
d_{0}+d_{1}+d_{2} \geqslant 1 / 2-a-2 \varepsilon^{\prime} .
$$

But $\{(\mathrm{D} 1),(\mathrm{D} 2),(\mathrm{D} 3),(\mathrm{D} 5),(\mathrm{D} 6)\}$ is an $(\varepsilon, 5)$-sufficient family.

Suppose secondly that $(s, t)=(3,1)$. Suppose that $A_{4}$ is the only set of type (c). We will prove that the following inequalities hold.

$$
\begin{aligned}
& d_{1}+d_{2} \geqslant 1 / 4-3 a / 2-3 \varepsilon^{\prime}, \\
& d_{3} \leqslant 1 / 4+3 a / 2+3 \varepsilon^{\prime} .
\end{aligned}
$$

Note that

$$
A_{4} \cap E \subseteq D_{1} \cup D_{2} \text { and } A_{4} \cap \mathrm{O} \subseteq D_{4} \cup D_{5} .
$$

Further, using Lemma 6.5 and Observation 6.6(i), we have

$$
d\left(A_{4} \cap J_{2}\right)=d\left(A_{4}\right)-d\left(A_{4} \backslash J_{2}\right) \geqslant\left(1 / 2-a-\varepsilon^{\prime}\right)-1 / n \geqslant 1 / 2-a-2 \varepsilon^{\prime} .
$$

Therefore

$$
\begin{gathered}
d_{1}+d_{2} \underset{(6.3),(6.9)}{\geqslant} d\left(\left(D_{1} \cup D_{2}\right) \cap J_{2}\right) \stackrel{(6.8)}{\geqslant} d\left(A_{4} \cap E \cap J_{2}\right) \geqslant d\left(A_{4} \cap J_{2}\right)-d\left(J_{2} \cap \mathrm{O}\right) \\
\left(1 / 2-a-2 \varepsilon^{\prime}\right)-\left(1 / 4+a / 2+\varepsilon^{\prime}\right)=1 / 4-3 a / 2-3 \varepsilon^{\prime},
\end{gathered}
$$

proving (D7). To prove (D8),

$$
\begin{aligned}
d_{3} & =d\left(D_{3} \cap J_{1}\right)+d\left(D_{3} \cap J_{2}\right) \\
& \leqslant d\left(J_{1}\right)-d\left(\left(D_{0} \cup D_{1}\right) \cap J_{1}\right)+d\left(J_{2}\right)-d\left(\left(D_{1} \cup D_{2} \cup D_{4} \cup D_{5}\right) \cap J_{2}\right) \\
& \quad(6.8) \\
& \leqslant 1-d\left(\left(D_{0} \cup D_{1}\right) \cap J_{1}\right)-d\left(A_{4} \cap J_{2}\right) \stackrel{(6.7),(6.9)}{\leqslant} 1-\left(1 / 4-a / 2-\varepsilon^{\prime}\right)-\left(1 / 2-a-2 \varepsilon^{\prime}\right) \\
& =1 / 4+3 a / 2+3 \varepsilon^{\prime},
\end{aligned}
$$

as desired. We have that $\{(\mathrm{D} 1),(\mathrm{D} 2),(\mathrm{D} 3),(\mathrm{D} 5),(\mathrm{D} 7),(\mathrm{D} 8)\}$ is an $(\varepsilon, 5)$-sufficient family. 
Case 3. $(s, t)=(2,3)$.

We will assume that $A_{1}$ and $A_{2}$ are type (b) sets (so $A_{1}=A_{2}=\mathrm{O}$ ), and $A_{3}, A_{4}, A_{5}$ are type (c) sets. Let $q_{i}:=d\left(A_{i} \cap J_{3}\right)$, for every $i \in\{3,4,5\}$. First, we will prove that the following constraints hold.

$$
\begin{aligned}
& d_{5} \geqslant 1 / 4-2 a-2 \varepsilon^{\prime}, \\
& d_{3} \leqslant 1 / 4+3 a+4 \varepsilon^{\prime} .
\end{aligned}
$$

We first prove (D9). By Lemma 6.7, we have

$$
\begin{aligned}
d_{5} & \geqslant d\left(D_{5} \cap I_{2}\right) \geqslant d\left(\mathrm{O} \cap\left(A_{3} \cap A_{4} \cap A_{5}\right) \cap I_{2}\right) \\
& \geqslant d\left(\left(A_{3} \cap A_{4} \cap A_{5}\right) \cap I_{2}\right)-d\left(E \cap I_{2}\right) \\
& \geqslant\left(1 / 2-2 a-\varepsilon^{\prime}\right)-\left(1 / 4+\varepsilon^{\prime}\right)=1 / 4-2 a-2 \varepsilon^{\prime},
\end{aligned}
$$

proving (D9). We now prove (D10). Notice that every element of $D_{3}$ lies in at least one type (c) set, i.e. $D_{3} \subseteq A_{3} \cup A_{4} \cup A_{5}$. So $\left|D_{3} \cap J_{1}\right| \leqslant \sum_{3 \leqslant i \leqslant 5}\left|A_{3} \cap J_{1}\right|=\sum_{3 \leqslant i \leqslant 5}\left|A_{3} \backslash J_{2}\right| \leqslant 3$ by Observation 6.6(i). Thus

$$
\begin{array}{lll}
d_{3} \underset{(6.3),(6.10)}{=} & d\left(D_{3} \cap J_{2}\right)+d\left(D_{3} \cap J_{1}\right) \leqslant d\left(J_{2}\right)-d\left(D_{5} \cap J_{2}\right)+3 / n \\
& \left(1 / 2+a+\varepsilon^{\prime}\right)-\left(1 / 4-2 a-2 \varepsilon^{\prime}\right)+3 / n \leqslant 1 / 4+3 a+4 \varepsilon^{\prime},
\end{array}
$$

as required.

Since $A_{1}=A_{2}=\mathrm{O}$, we have $E \subseteq D_{0} \cup \ldots \cup D_{3}$. Let $B:=J_{3} \cap E \cap\left(D_{2} \cup D_{3}\right)$. Assume now that $d(B) \leqslant a / 4$. We claim that, in this case, the following hold:

$$
\begin{aligned}
d_{0}+d_{1} & \geqslant 1 / 4-a / 4-2 \varepsilon^{\prime} . \\
d_{2} & \geqslant 1 / 4-a / 2-\varepsilon^{\prime} .
\end{aligned}
$$

Indeed, to see the first inequality, observe that

$$
d_{0}+d_{1} \geqslant d\left(D_{0} \cap J_{1}\right)+d\left(J_{3} \cap E\right)-\frac{a}{4} \stackrel{(6.6)}{\geqslant}\left(\frac{1}{4}-\frac{a}{2}-\varepsilon^{\prime}\right)+\frac{\left\lfloor\left|J_{3}\right| / 2\right\rfloor}{n}-\frac{a}{4} \geqslant \frac{1}{4}-\frac{a}{4}-2 \varepsilon^{\prime} .
$$

The second is a consequence of (6.6). But $\{(\mathrm{D} 1),(\mathrm{D} 2),(\mathrm{D} 3),(\mathrm{D} 9),(\mathrm{D} 10),(\mathrm{D} 11),(\mathrm{D} 12)\}$ is an $(\varepsilon, 5)$-sufficient family.

The only case left is when $d(B)>a / 4$. We claim that, in this case, the following hold:

$$
\begin{aligned}
& d_{2} \geqslant 1 / 4-a / 4-2 \varepsilon^{\prime} . \\
& d_{0} \geqslant 1 / 4-a / 2-\varepsilon^{\prime} .
\end{aligned}
$$

The second inequality is simply (6.6). To see why the first holds, let $m:=\min (B)$. Then

$$
m<\left\lfloor\frac{n}{2}\right\rfloor-2 \cdot\left(\frac{a n}{4}-1\right) \leqslant \frac{n}{2}-\frac{a n}{2}+2 .
$$


Since $m \in E$, we know that $m \notin A_{1} \cup A_{2}$. In addition, since $m \in D_{2} \cup D_{3}$, without loss of generality, we can assume that $m \in A_{3} \cap A_{4}$. Therefore, by the definition of type (c) sets, we see that $\left|A_{3}\right|,\left|A_{4}\right| \leqslant m$. Thus $\left|A_{3}\right|+\left|A_{4}\right|<n-a n+4$. So, recalling that $\left|A_{1}\right|=\left|A_{2}\right|=$ $\lceil n / 2\rceil$, the definition of $a$ implies that $\left|A_{5}\right|=3\lceil n / 2\rceil-$ an $-\left|A_{3}\right|-\left|A_{4}\right|>n / 2-4$. Thus $\min \left(A_{5}\right)>n / 2-4$. So $\left|A_{5} \cap J_{3}\right| \leqslant 3$. But $E \cap D_{3} \subseteq A_{3} \cap A_{4} \cap A_{5}$, so $\left|J_{3} \cap E \cap D_{3}\right| \leqslant 3$. Since $d(B)>a / 4$, we have $d\left(J_{3} \cap E \cap D_{2}\right)>a / 4-3 / n$. Thus

$$
d_{2} \geqslant d\left(J_{1} \cap D_{2}\right)+d\left(J_{3} \cap E \cap D_{2}\right) \stackrel{(6.6)}{\geqslant} 1 / 4-a / 2-\varepsilon^{\prime}+a / 4-3 / n=1 / 4-a / 4-2 \varepsilon^{\prime},
$$

as required. Now $\{(\mathrm{D} 1),(\mathrm{D} 2),(\mathrm{D} 3),(\mathrm{D} 9),(\mathrm{D} 10),(\mathrm{D} 13),(\mathrm{D} 14)\}$ is an $(\varepsilon, 5)$-sufficient family.

Case $4 .(s, t)=(3,2)$.

We will assume that $A_{1}, A_{2}$ and $A_{3}$ are type (b) sets (and so $A_{4}$ and $A_{5}$ are type (c) sets). We will prove that the following constraints hold.

$$
\begin{aligned}
& d_{0} \geqslant 1 / 4-a / 2-\varepsilon^{\prime} . \\
& d_{2}+d_{5} \geqslant 1 / 2-2 a-\varepsilon^{\prime}, \\
& d_{1}+d_{3}+d_{4} \leqslant 1 / 4+5 a / 2+3 \varepsilon^{\prime}, \\
& d_{2} \leqslant 1 / 4+a / 2+\varepsilon^{\prime} .
\end{aligned}
$$

The first inequality (D15) is simply (6.6). For (D16), observe that $E \cap A_{4} \cap A_{5} \subseteq D_{2}$, and $\mathrm{O} \cap A_{4} \cap A_{5}=D_{5}$. So

$$
A_{4} \cap A_{5} \subseteq D_{2} \cup D_{5} \quad \text { and } \quad d_{2}+d_{5}=d\left(D_{2} \cup D_{5}\right) \geqslant d\left(A_{4} \cap A_{5}\right) \geqslant 1 / 2-2 a-\varepsilon^{\prime},
$$

where we used Lemma 6.5 for the final inequality. For (D17), we have

$$
\begin{aligned}
d_{1}+d_{3}+d_{4} & \leqslant d\left(\left(D_{1} \cup D_{3} \cup D_{4}\right) \cap J_{1}\right)+d\left(\left(D_{1} \cup D_{3} \cup D_{4}\right) \cap J_{2}\right) \\
& \leqslant d\left(J_{1}\right)-d\left(D_{0} \cap J_{1}\right)+d\left(J_{2} \backslash\left(A_{4} \cap A_{5}\right)\right) \\
& =d\left(J_{1}\right)-d\left(D_{0} \cap J_{1}\right)+d\left(J_{2}\right)-d\left(A_{4} \cap A_{5}\right)+d\left(\left(A_{4} \cap A_{5}\right) \backslash J_{2}\right) \\
& \leqslant 1-\left(1 / 4-a / 2-\varepsilon^{\prime}\right)-\left(1 / 2-2 a-\varepsilon^{\prime}\right)+1 / n=1 / 4+5 a / 2+3 \varepsilon^{\prime} .
\end{aligned}
$$

The final inequality follows from (6.6), (6.11) and Observation 6.6(i).

Finally we will prove (D18). Observe that $E \backslash\left(A_{4} \cup A_{5}\right) \subseteq D_{0}$ and $\mathrm{O} \backslash\left(A_{4} \cup A_{5}\right) \subseteq D_{3}$. By Observation 6.6(i), we have that $\left|J_{1} \cap\left(A_{4} \cup A_{5}\right)\right|=\left|\left(A_{4} \cup A_{5}\right) \backslash J_{2}\right| \leqslant 2$. Therefore $\mid\left(J_{1} \cap\left(D_{0} \cup D_{3}\right)|\geqslant| J_{1} \mid-2\right.$ and so $\left|D_{2} \cap J_{1}\right| \leqslant 2$. Further, $\mathrm{O} \subseteq D_{3} \cup D_{4} \cup D_{5}$. In particular, $D_{2} \cap J_{2} \subseteq E \cap J_{2}$. Combining these facts, we see that

$$
d_{2}=d\left(D_{2} \cap J_{1}\right)+d\left(D_{2} \cap J_{2}\right) \leqslant 2 / n+d\left(E \cap J_{2}\right) \leqslant 2 / n+\frac{\left\lceil\left|J_{2}\right| / 2\right\rceil}{n} \stackrel{(6.3)}{\leqslant} 1 / 4+a / 2+\varepsilon^{\prime},
$$

as desired. But $\{(\mathrm{D} 1),(\mathrm{D} 2),(\mathrm{D} 3),(\mathrm{D} 15),(\mathrm{D} 16),(\mathrm{D} 17),(\mathrm{D} 18)\}$ is an $(\varepsilon, 5)$-sufficient family.

Case $5 .(s, t)=(2,2)$ 
We will assume that $A_{1}, A_{2}$ are of type (b); $A_{3}, A_{4}$ are of type (c); and $A_{5}$ is of type (a). Our immediate aim is to prove that the following inequalities hold.

$$
\begin{aligned}
& d_{0}+d_{1} \geqslant 1 / 4-a / 2-\varepsilon^{\prime} . \\
& d_{3}-d_{2} \leqslant 1 / 4+4 a+6 \varepsilon^{\prime} .
\end{aligned}
$$

The first is a consequence of (6.7). We will now prove (D20). This requires careful analysis of the small unstructured set $A_{5}$. Define sets

$$
\begin{aligned}
& I_{1}^{\prime}:=\left[1,\left\lceil\frac{n}{4}\right\rceil\right], \quad I_{1}^{\prime \prime}:=J_{1} \backslash I_{1}^{\prime}=\left[\left\lceil\frac{n}{4}\right\rceil+1,\left\lfloor\frac{n}{2}\right\rfloor-a n\right], \\
& X_{1}:=A_{5} \cap O \cap I_{1}^{\prime}, \quad X_{2}:=A_{5} \cap O \cap I_{1}^{\prime \prime}, \\
& x_{i}:=d\left(X_{i}\right) \text { for } i=1,2 ; \\
& Y_{o}:=A_{5} \cap O \cap J_{2}, \quad Y_{e}:=A_{5} \cap E \cap J_{2} \text {, } \\
& y_{o}:=d\left(Y_{o}\right) \text { and } y_{e}:=d\left(Y_{e}\right) \text {, } \\
& S:=\left\{x+y: x, y \in X_{2} \cup\left(Y_{o} \cap J_{3}\right)\right\} .
\end{aligned}
$$

Clearly, it suffices to show that

$$
\begin{aligned}
& d_{3} \leqslant 3 / 8+7 a / 2-x_{2}+5 \varepsilon^{\prime} \quad \text { and } \\
& d_{2} \geqslant 1 / 8-a / 2-x_{2}-\varepsilon^{\prime} .
\end{aligned}
$$

Let $Z:=J_{1} \cap\left(A_{3} \cup A_{4}\right)$. By Observation 6.6(i), we have $|Z| \leqslant 2$. To prove (6.12), we bound $d\left(D_{3} \cap J_{1}\right)$ and $d\left(D_{3} \cap J_{2}\right)$ separately. If $v \in D_{3} \cap E$, then $v \in A_{3} \cap A_{4} \cap A_{5}$. So $D_{3} \cap J_{1} \cap E \subseteq Z$. Similarly, $D_{3} \cap J_{1} \cap \mathrm{O} \subseteq\left(A_{5} \cap J_{1}\right) \cup Z$. Thus

$$
d\left(D_{3} \cap J_{1}\right)=d\left(D_{3} \cap J_{1} \cap E\right)+d\left(D_{3} \cap J_{1} \cap O\right) \leqslant d(Z)+x_{1}+x_{2} \leqslant x_{1}+x_{2}+\varepsilon^{\prime} .
$$

Now, $A_{3} \cap A_{4} \cap \mathrm{O} \subseteq D_{4} \cup D_{5}$. Further, $D_{3} \cap E \subseteq A_{5}$. Therefore

$$
\begin{aligned}
d\left(D_{3} \cap J_{2}\right) & =d\left(D_{3} \cap J_{2} \cap E\right)+d\left(D_{3} \cap J_{2} \cap O\right) \leqslant y_{e}+d\left(J_{2} \cap O\right)-d\left(A_{3} \cap A_{4} \cap J_{2} \cap O\right) \\
& \leqslant y_{e}+d\left(J_{2} \cap O\right)-\left(d\left(A_{3} \cap A_{4} \cap J_{2}\right)-d\left(J_{2} \cap E\right)\right) \\
& \leqslant y_{e}+d\left(J_{2}\right)-d\left(A_{3} \cap A_{4} \cap I_{2}\right) \leqslant y_{e}+\left(1 / 2+a+\varepsilon^{\prime}\right)-\left(1 / 2-2 a-2 \varepsilon^{\prime}\right) \\
& =y_{e}+3 a+3 \varepsilon^{\prime},
\end{aligned}
$$

where we used (6.3) and Lemma 6.7 for the final inequality.

For every $s \in S$, we have that $s$ is even and at most $n$, and additionally $s \geqslant 2(\lceil n / 4\rceil+$ $1) \geqslant\lfloor n / 2\rfloor-a n+1$. Thus $S \subseteq J_{2} \cap E$. Since $A_{5}$ is sum-free, we have that $Y_{e} \cap S=\emptyset$. So $|S|+\left|Y_{e}\right| \leqslant\left|J_{2} \cap E\right|$. By Proposition 2.4 applied to $S$, we have $|S| \geqslant 2\left|X_{2} \cup\left(Y_{o} \cap J_{3}\right)\right|-1$. Thus

$$
2\left(\left|X_{2}\right|+\left|Y_{o} \cap J_{3}\right|\right)+\left|Y_{e}\right| \leqslant|S|+\left|Y_{e}\right|+1 \leqslant\left|J_{2} \cap E\right|+1 \stackrel{(6.3)}{\leqslant}\left(1 / 4+a / 2+\varepsilon^{\prime}\right) n .
$$

Combining this with (6.14) and (6.15) we have

$$
\begin{aligned}
d_{3} & \leqslant x_{1}+x_{2}+y_{e}+3 a+4 \varepsilon^{\prime} \stackrel{(6.16)}{\leqslant} x_{1}+\left(1 / 4+a / 2+\varepsilon^{\prime}-x_{2}\right)+3 a+4 \varepsilon^{\prime} \\
& \leqslant 3 / 8+7 a / 2-x_{2}+5 \varepsilon^{\prime},
\end{aligned}
$$


where we used the trival bound $x_{1} n \leqslant\left\lceil\left|I_{1}^{\prime}\right| / 2\right\rceil$. This finishes the proof of (6.12).

For (6.13), notice that $\mathrm{O} \backslash\left(A_{3} \cup A_{4} \cup A_{5}\right) \subseteq D_{2}$. By definition, $J_{1} \backslash Z$ is disjoint from $A_{3} \cup A_{4}$. Therefore

$$
\begin{aligned}
d_{2} & \geqslant d\left(\mathrm{O} \cap I_{1}^{\prime \prime}\right)-d\left(\mathrm{O} \cap I_{1}^{\prime \prime} \cap\left(A_{3} \cup A_{4}\right)\right)-d\left(\mathrm{O} \cap I_{1}^{\prime \prime} \cap A_{5}\right) \\
& \geqslant d\left(\mathrm{O} \cap I_{1}^{\prime \prime}\right)-d(Z)-x_{2} \geqslant \frac{1}{n}\left\lfloor\left|I_{1}^{\prime \prime}\right| / 2\right\rfloor-2 / n-x_{2} \geqslant 1 / 8-a / 2-x_{2}-\varepsilon^{\prime},
\end{aligned}
$$

as required. We have proved (6.13) and hence (D20).

The remainder of the proof will be divided into two final subcases. First, suppose that $Y_{o} \subseteq J_{3}$. Now (6.16) implies that $2\left(x_{2}+y_{o}\right)+y_{e} \leqslant 1 / 4+a / 2+\varepsilon^{\prime}$. Thus

$$
d\left(A_{5}\right)=x_{1}+x_{2}+y_{o}+y_{e} \leqslant d\left(\mathrm{O} \cap I_{1}^{\prime}\right)+1 / 4+a / 2+\varepsilon^{\prime} \leqslant 3 / 8+a / 2+2 \varepsilon^{\prime} .
$$

Lemma 6.3 implies that $\sum_{i \in[4]} d\left(A_{i}\right) \leqslant 2-a+\varepsilon^{\prime}$. Adding these, we see that

$$
\sum_{i \in[5]} i d_{i}=\sum_{i \in[5]} d\left(A_{i}\right) \leqslant 19 / 8-a / 2+3 \varepsilon^{\prime}
$$

Now $\{(\mathrm{D} 1),(\mathrm{D} 3),(\mathrm{D} 19),(\mathrm{D} 20),(\mathrm{D} 21)\}$ is an $(\varepsilon, 5)$-sufficient family.

The final subcase is when $Y_{o} \backslash J_{3} \neq \emptyset$. Let $w \in Y_{o} \backslash J_{3}$. So $w \geqslant\lfloor n / 2\rfloor+1$. We claim that the following inequality holds:

$$
d_{3} \leqslant 5 / 16+7 a / 2+5 \varepsilon^{\prime}
$$

To prove this, define

$$
X_{1}^{\prime}:=X_{1} \backslash\left\{\left\lceil\frac{n}{4}\right\rceil\right\} \quad \text { and } \quad D:= \begin{cases}\left\{w+x: x \in X_{1}^{\prime}\right\} & \text { if } w \in\left[\left\lfloor\frac{n}{2}\right\rfloor+1, n-\left\lceil\frac{n}{4}\right\rceil\right], \\ \left\{w-x: x \in X_{1}^{\prime}\right\} & \text { if } w \in\left[n-\left\lceil\frac{n}{4}\right\rceil+1, n\right] .\end{cases}
$$

Now, $w \in \mathrm{O}$ and $X_{1}^{\prime} \subseteq \mathrm{O}$, so every element of $D$ is even. In both cases it is easy to check that $D \subseteq E \cap J_{2}$. Since $A_{5}$ is sum-free, $D \cap Y_{e}=\emptyset$, and so $D$ and $Y_{e}$ are disjoint subsets of $E \cap J_{2}$. In particular,

$$
x_{1}+y_{e} \leqslant|D| / n+y_{e}+1 / n \leqslant d\left(J_{2} \cap E\right)+1 / n \stackrel{(6.3)}{\leqslant} 1 / 4+a / 2+\varepsilon^{\prime} .
$$

This then implies that

$$
\begin{aligned}
d_{3} & =d\left(D_{3} \cap J_{1}\right)+d\left(D_{3} \cap J_{2}\right) \stackrel{(6.14),(6.15)}{\leqslant} x_{1}+x_{2}+y_{e}+3 a+4 \varepsilon^{\prime} \\
& =\frac{1}{2}\left(x_{1}+y_{e}\right)+\frac{1}{2}\left(2 x_{2}+y_{e}\right)+\frac{x_{1}}{2}+3 a+4 \varepsilon^{\prime} \stackrel{(6.16),(6.18)}{\leqslant} \frac{1}{4}+\frac{a}{2}+\frac{x_{1}}{2}+3 a+5 \varepsilon^{\prime} \\
& \leqslant \frac{5}{16}+\frac{7 a}{2}+5 \varepsilon^{\prime},
\end{aligned}
$$

This proves the claim. But $\{(\mathrm{D} 1),(\mathrm{D} 2),(\mathrm{D} 3),(\mathrm{D} 19),(\mathrm{D} 20),(\mathrm{D} 22)\}$ is an $(\varepsilon, 5)$-sufficient family. This completes the proof of the final case, and hence completes the proof of Lemma 6.2. 


\section{Concluding remarks}

We determined $f(n, r)$ exactly when $r=2$ (Theorem 1.2). It would be interesting to proceed from our stability result (Theorem 1.3) and obtain an exact result for $r=3$, and characterise the extremal sets. It seems possible to extract a statement about stability from the proof of Theorem 1.4 by more careful analysis of the linear programs. That is, the following may be obtainable. For all $\varepsilon>0$, as long as $n$ is a sufficiently large integer: if $r=4$ and $A \subseteq[n]$ is extremal, then one of $A \triangle \mathrm{O}, A \triangle I_{2}$ and $A \triangle\left(\mathrm{O} \cup I_{2}\right)$ has size at most $\varepsilon n$; and if $r=5$ and $A \subseteq[n]$ is extremal, then $\left|A \triangle\left(\mathrm{O} \cup I_{2}\right)\right| \leqslant \varepsilon n$.

It is also possible that the method used to prove Theorem 1.4 (namely finding sufficient linear constraints) can prove the analogous result for $r=6$. The main obstacle is the fact that, among extremal $A_{1}, \ldots, A_{6}$, one cannot a priori guarantee less than two type (a) sets. This leads to 18 different values of $(s, t)$ to consider. Since the proof for $r=5$ was already very involved, we did not pursue this further.

Finally, for large $r$, the value of $f(n, r)$ and the structure of the extremal sets is completely open.

\section{Acknowledgements}

We are grateful to Tuan Tran for helpful discussions, and to the anonymous referee whose detailed comments improved the presentation of the paper.

\section{References}

[1] N. Alon, J. Balogh, P. Keevash and B. Sudakov, The number of edge colorings with no monochromatic cliques, J. London Math. Soc. 70 (2004), 273-288.

[2] N. Alon and R. Yuster, The number of orientations having no fixed tournament, Combinatorica 26 (2006), 1-16.

[3] J. Balogh, A remark on the number of edge colorings of graphs, Europ. J. Comb. 27 (2006), 565-573.

[4] J. Balogh, R. Morris and W. Samotij, Independent sets in hypergraphs, J. Amer. Math. Soc. 28 (2015), 669-709.

[5] J. Balogh, H. Liu, M. Sharifzadeh and A. Treglown, The number of maximal sum-free subsets of integers, Proc. Amer. Math. Soc., 143, (2015) 4713-4721.

[6] F. S. Benevides, C. Hoppen and R. M. Sampaio, Edge-colorings of graphs avoiding complete graphs with a prescibed coloring, 340 (9) (2017), 2143-2160.

[7] P. Cameron and P. Erdős, On the number of sets of integers with various properties, in Number Theory (R.A. Mollin, ed.), 61-79, Walter de Gruyter, Berlin, 1990.

[8] D. Clemens, S. Das and T. Tran, Colourings without monochromatic disjoint pairs, European J. Combin. 70 (2018), 99-124. 
[9] J. Deshouillers, G. Freiman, V. Sós and M. Temkin, On the structure of sum-free sets II, Astérisque 258 (1999), 149-161.

[10] P. Erdős, Some new applications of probability methods to combinatorial analysis and graph theory, Proceedings of the Fifth Southeastern Conference on Combinatorics, Graph Theory and Computing, Congress Numerantium X (1974), 39-51.

[11] P. Erdős, Some of my favorite problems in various branches of combinatorics, Matematiche (Catania) 47 (1992), 231-240.

[12] P. Erdős, D.J. Kleitman and B.L. Rothschild, Asymptotic enumeration of $K_{n}$-free graphs, in Colloq. Int. Teorie Combin. (Rome, 1973), Tomo II, Atti dei Convegni Lincei, No. 17, Accademia Nazionale dei Lincei, Rome, 1976, 19-27.

[13] J. Fox, A new proof of the graph removal lemma, Ann. of Math., 174 (2) (2011), $561-579$.

[14] B. Green, The Cameron-Erdős conjecture, Bull. London Math. Soc. 36 (2004), 769-778.

[15] B. Green, A Szemerédi-type regularity lemma in abelian groups, with applications, Geom. Funct. Anal. 15 (2005), 340-376.

[16] B. Green and I. Ruzsa, Counting sumsets and sum-free sets modulo a prime, Studia Sci. Math. Hungarica 41 (2004), 285-293.

[17] B. Green and I. Ruzsa, Sum-free sets in abelian groups, Israel J. Math 147 (2005), 157-189.

[18] H. Hàn and A. Jiménez, Maximum number of sum-free colorings in finite abelian groups, Israel J. Math 226 (2018), 505-534.

[19] C. Hoppen, Y. Kohayakawa and H. Lefmann, Kneser colorings of uniform hypergraphs, Elec. Notes in Disc. Math. 34 (2009), 219-223.

[20] C. Hoppen, Y. Kohayakawa and H. Lefmann, Edge colourings of graphs avoiding monochromatic matchings of a given size, Comb. Prob. Comp. 21 (2012), 203-218.

[21] C. Hoppen, Y. Kohayakawa and H. Lefmann, Edge-colorings of graphs avoiding fixed monochromatic subgraphs with linear Turán number, Europ. J. Comb. 35 (2014), 354-373.

[22] C. Hoppen and H. Lefmann, Edge-colorings avoiding a fixed matching with a prescribed color pattern, Europ. J. Comb. 47 (2015), 75-94.

[23] C. Hoppen, H. Lefmann and K. Odermann, A coloring problem for intersecting vector spaces, Disc. Math. 339 (2016), 2941-2954.

[24] C. Hoppen, H. Lefmann, K. Odermann and J. Sanches, Edge-colorings avoiding fixed rainbow stars, Elec. Notes in Disc. Math. 50 (2015), 275-280.

[25] D. Kleitman and K.J. Winston, The asymptotic number of lattices, Ann. Discrete Math. 6 (1980), 243-249, Combinatorial mathematics, optimal designs and their applications (Proc. Sympos. Combin. Math. and Optimal Design, Colorado State Univ., Fort Collins, Colo., 1978). 
[26] D. Kleitman and K.J. Winston, On the number of graphs without 4-cycles, Discrete Math. 6 (1982), 167-172.

[27] D. Král', O. Serra and L. Vena, A combinatorial proof of the Removal Lemma for groups, J. Combin. Theory A 116, (2009), 971-978.

[28] H. Lefmann, Y. Person, V. Rödl and M. Schacht, On colorings of hypergraphs without monochromatic Fano planes, Comb. Prob. Comp. 18 (2009), 803-818.

[29] H. Lefmann, Y. Person and M. Schacht, A structural result for hypergraphs with many restricted edge colorings, J. Comb. 1 (2010), 441-475.

[30] W. Mantel, Problem 28, Wiskundige Opgaven 10 (1907), 60-61.

[31] O. Pikhurko, K. Staden and Z. Yilma, The Erdős-Rothschild problem on edgecolourings with forbidden monochromatic cliques, Math. Proc. Cambridge Phil. Soc 163 (2017), 341-356.

[32] O. Pikhurko and Z. Yilma, The maximum number of $K_{3}$-free and $K_{4}$-free edge 4colorings, J. London Math. Soc. 85 (2012), 593-615.

[33] I.Z. Ruzsa and E. Szemerédi, Triple systems with no six points carrying three triangles, in Combinatorics (Keszthely, 1976), Coll. Math. Soc. J. Bolyai 18, Volume II, 939-945.

[34] A.A. Sapozhenko, The Cameron-Erdős conjecture, (Russian) Dokl. Akad. Nauk., 393, (2003), 749-752.

[35] D. Saxton and A. Thomason, Hypergraph containers, Invent. Math. 201 (2015), 925-992.

[36] E. Szemerédi, 'Regular partitions of graphs' in Proc. Colloq. Int. CNRS, Paris, 1976, pp. 309-401.

[37] T. Tran, On the structure of large sum-free sets in integers, Israel J. Math. 228, (2018), 249-292.

[38] R. Yuster, The number of edge colorings with no monochromatic triangle, J. Graph Theory 21 (1996), 441-452.

\section{A Appendix}

It remains to prove that the families obtained in the proof of Lemma 6.2 are indeed $(\varepsilon, r)$-sufficient. Namely, we require that the following lemma holds.

Lemma A.1. Given $\varepsilon>0$, for $r \in\{4,5\}$ there exists $\delta, n_{0}>0$ such that whenever $\delta \leqslant \varepsilon^{\prime} \leqslant 1 / 100$ is a real constant and $n \geqslant n_{0}$ is an integer and $A_{1}, \ldots, A_{r}$ are maximal sum-free subsets of $[n]$, we have that: The following families (depending on $\varepsilon^{\prime}$ ) are $(\varepsilon, 4)$ - 
sufficient.
1) $\quad\left\{\left(\mathrm{C} 0^{*}\right),(\mathrm{C} 1)\right\}$
(Case 0)
2) $\quad\{(\mathrm{C} 1),(\mathrm{C} 2),(\mathrm{C} 4),(\mathrm{C} 5)\}$
(Case 1)
3)
$\{(\mathrm{C} 1),(\mathrm{C} 2),(\mathrm{C} 3),(\mathrm{C} 4),(\mathrm{C} 6)\}$
(Case 1)
4) $\quad\{(\mathrm{C} 1),(\mathrm{C} 2),(\mathrm{C} 3),(\mathrm{C} 4),(\mathrm{C} 7)\}$
(Case 2)
$5)$
$\{(\mathrm{C} 1),(\mathrm{C} 2),(\mathrm{C} 3),(\mathrm{C} 8),(\mathrm{C} 9)\}$
(Case 3)

The following families (depending on $\varepsilon^{\prime}$ ) are $(\varepsilon, 5)$-sufficient.

6) $\quad\left\{\left(\mathrm{D} 0^{*}\right),(\mathrm{D} 1)\right\}$

(Case 0)

7) $\quad\{(\mathrm{D} 1),(\mathrm{D} 2),(\mathrm{D} 3),(\mathrm{D} 4)\}$

(Case 1)

8) $\quad\{(\mathrm{D} 1),(\mathrm{D} 2),(\mathrm{D} 3),(\mathrm{D} 5),(\mathrm{D} 6)\}$

(Case 2)

9) $\quad\{(\mathrm{D} 1),(\mathrm{D} 2),(\mathrm{D} 3),(\mathrm{D} 5),(\mathrm{D} 7),(\mathrm{D} 8)\}$

(Case 2)

10) $\quad\{(\mathrm{D} 1),(\mathrm{D} 2),(\mathrm{D} 3),(\mathrm{D} 9),(\mathrm{D} 10),(\mathrm{D} 11),(\mathrm{D} 12)\}$

(Case 3)

11)

$\{(\mathrm{D} 1),(\mathrm{D} 2),(\mathrm{D} 3),(\mathrm{D} 9),(\mathrm{D} 10),(\mathrm{D} 13),(\mathrm{D} 14)\}$

(Case 3)

12) $\quad\{(\mathrm{D} 1),(\mathrm{D} 2),(\mathrm{D} 3),(\mathrm{D} 15),(\mathrm{D} 16),(\mathrm{D} 17),(\mathrm{D} 18)\}$

(Case 4)

13) $\quad\{(\mathrm{D} 1),(\mathrm{D} 3),(\mathrm{D} 19),(\mathrm{D} 20),(\mathrm{D} 21)\}$

(Case 4)

14)

$\{(\mathrm{D} 1),(\mathrm{D} 2),(\mathrm{D} 3),(\mathrm{D} 19),(\mathrm{D} 20),(\mathrm{D} 22)\}$

(Case 5).

Proof. For 1),3) and 4), taking $\varepsilon^{\prime}=1 / 100$ in Mathematica yields $\sum_{i \in[4]} d_{i} \log i<1-$ $1 / 1000$, so we are done in these cases. Given a linear maximisation (primal) program:

Maximise $\boldsymbol{c}^{\top} \boldsymbol{d}$ subject to $A \boldsymbol{d} \leqslant \boldsymbol{b}$ and $\boldsymbol{d} \geqslant \mathbf{0}$,

the dual minimisation program is:

Minimise $\boldsymbol{b}^{\top} \boldsymbol{y}$ subject to $A^{\top} \boldsymbol{y} \geqslant \boldsymbol{c}$ and $\boldsymbol{y} \geqslant \mathbf{0}$.

Family 2) Taking the program represented by 2) as the primal, we have

$$
\begin{aligned}
& \begin{array}{llllll}
\text { a } & d_{0} & d_{1} & d_{2} & d_{3} & d_{4}
\end{array}
\end{aligned}
$$

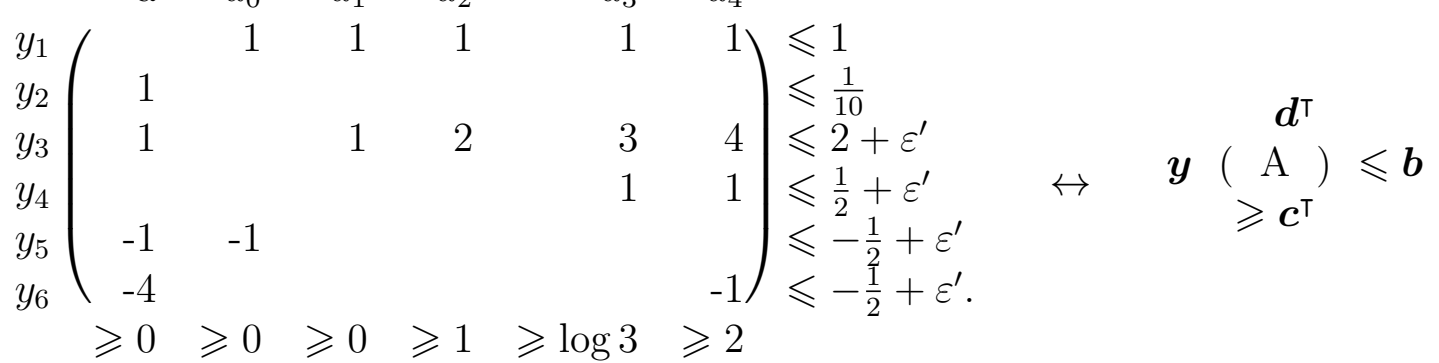

A feasible solution to the dual program is $\boldsymbol{y}^{*}=\left(0,0, \frac{\log 3}{3}, 0,0, \frac{4 \log 3}{3}-2\right)$. The objective function value of the dual at $\boldsymbol{y}^{*}$ is

$\boldsymbol{b}^{\top} \boldsymbol{y}^{*}=\left(2+\varepsilon^{\prime}\right) \frac{\log 3}{3}+\left(-\frac{1}{2}+\varepsilon^{\prime}\right)\left(\frac{4 \log 3}{3}-2\right)=1+\left(\frac{5 \log 3}{3}-2\right) \varepsilon^{\prime} \leqslant 1+\varepsilon^{\prime} \leqslant 1+\varepsilon$. 
By the weak duality theorem, any feasible solution $\boldsymbol{x}$ to the primal maximisation linear program satisfies $\boldsymbol{c}^{\top} \boldsymbol{x} \leqslant \boldsymbol{b}^{\top} \boldsymbol{y}^{*} \leqslant 1+\varepsilon$. Thus the family in 2) is ( $\left.\varepsilon, 4\right)$-sufficient.

Family 5) The family yields the following primal and dual linear programs.

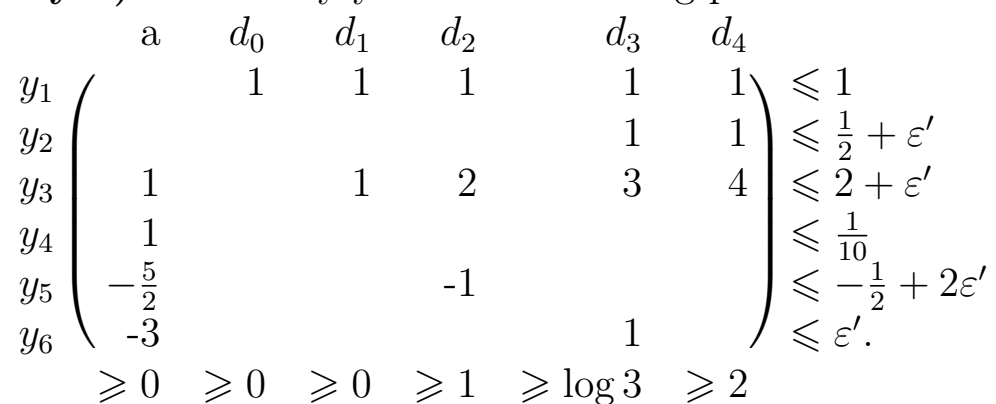

A feasible solution to the dual program is $\boldsymbol{y}^{*}=\left(0,0, \frac{1}{2}, 0,0, \log 3-\frac{3}{2}\right)$. The objective function value of the dual at $\boldsymbol{y}^{*}$ is

$$
\boldsymbol{b}^{\top} \boldsymbol{y}^{*}=\left(2+\varepsilon^{\prime}\right) \cdot \frac{1}{2}+\varepsilon^{\prime}\left(\log 3-\frac{3}{2}\right)=1+(\log 3-1) \varepsilon^{\prime} \leqslant 1+\varepsilon^{\prime} \leqslant 1+\varepsilon .
$$

We are again done by the weak duality theorem.

Now we let $r=5$ and consider families 6)-14). For 6)-9), 13) and 14), taking $\varepsilon^{\prime}=1 / 100$ in Mathematica yields $\sum_{i \in[5]} d_{i} \log i<\frac{1}{4} \log 30-1 / 10^{4}$, so we are done in these cases. It remains to consider 10)-12).

Family 10) The family yields the following primal and dual linear programs.

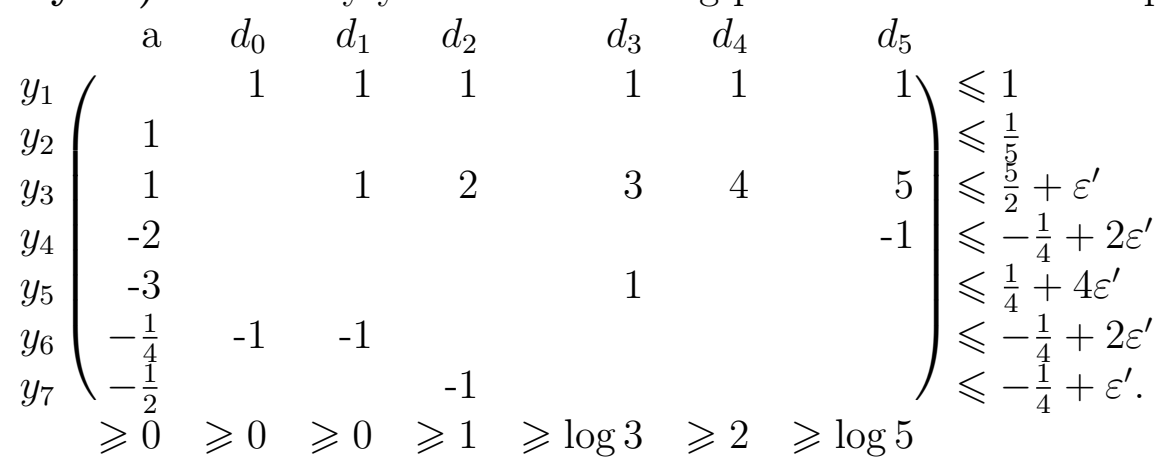

A feasible solution to the dual program is

$$
\boldsymbol{y}^{*}=\left(4 x, 0, \frac{1}{2}-x, \frac{5}{2}-\log 5-x, \log 3-\frac{3}{2}-x, 4 x, 2 x\right)
$$

where $x=\frac{3}{2} \log 3-\log 5$. The objective function value of the dual at $\boldsymbol{y}^{*}$ is

$$
\boldsymbol{b}^{\top} \boldsymbol{y}^{*}=\frac{1}{4} \log 30+\left(-\frac{1}{2}+22 \log 3-14 \log 5\right) \varepsilon^{\prime}<\frac{1}{4} \log 30+2 \varepsilon^{\prime} \leqslant \frac{1}{4} \log 30+\varepsilon \text {. }
$$

So the family in 10$)$ is $(\varepsilon, 5)$-sufficient. 
Family 11) The family yields the following primal and dual linear programs.

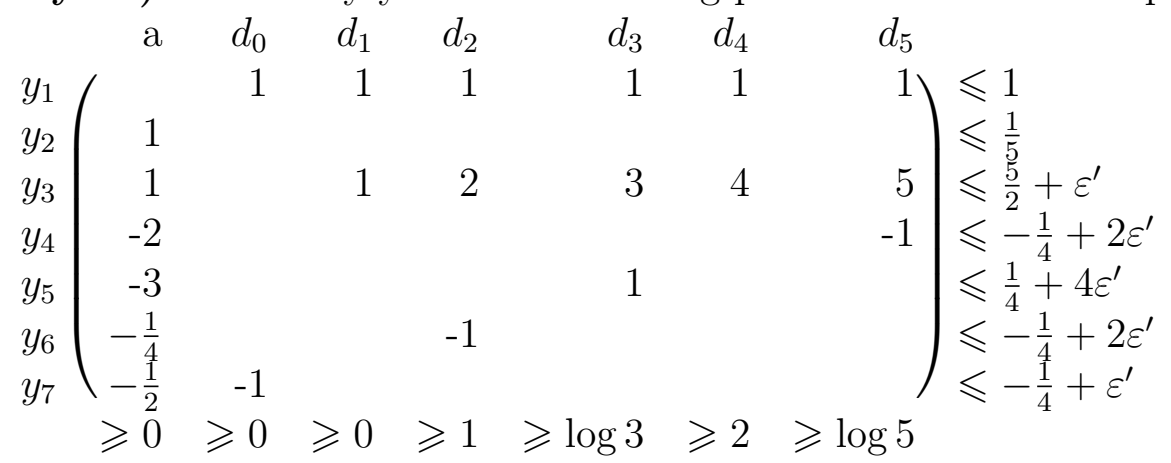

A feasible solution to the dual is

$$
\boldsymbol{y}^{*}=\left(4 x, 0, \frac{1}{2}-x, \frac{5}{2}-\log 5-x, \log 3-\frac{3}{2}-x, 4 x, 4 x\right)
$$

where $x=2 \log 3-\frac{4}{3} \log 5$. The objective function value of the dual at $\boldsymbol{y}^{*}$ is

$$
\boldsymbol{b}^{\top} \boldsymbol{y}^{*}=\frac{1}{4} \log 30+\left(-\frac{1}{2}+6 \log 3-\frac{10}{3} \log 5\right) \varepsilon^{\prime}<\frac{1}{4} \log 30+2 \varepsilon^{\prime} \leqslant \frac{1}{4} \log 30+\varepsilon .
$$

So the family in 11$)$ is $(\varepsilon, 5)$-sufficient.

Family 12) The family yields the following primal and dual linear programs.

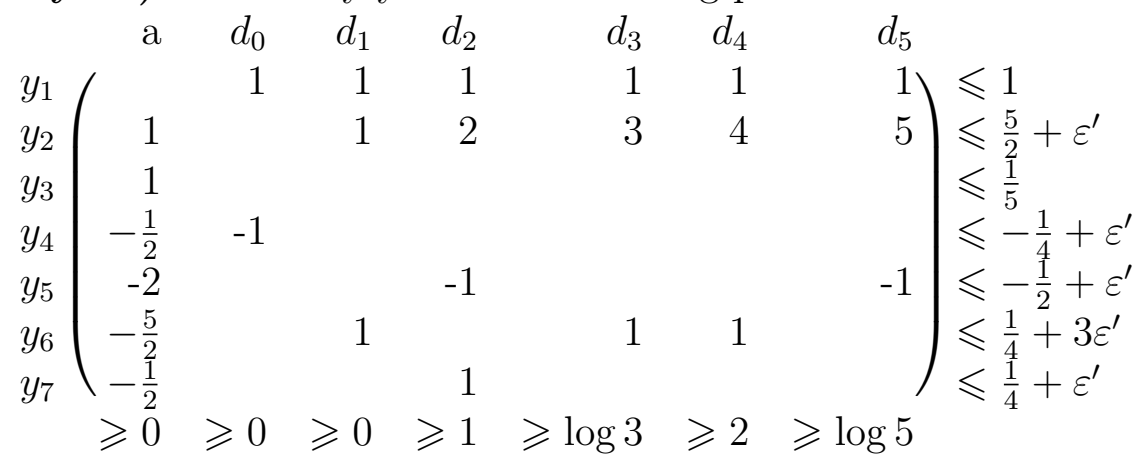

A feasible solution to the dual is

$$
\boldsymbol{y}^{*}=\left(y_{1}, \frac{\log 5}{5}-\frac{y_{1}}{5}, 0, y_{1}, 0, \log 3-\frac{3}{5} \log 5-\frac{2}{5} y_{1}, 1-\frac{2}{5} \log 5-\frac{3}{5} y_{1}\right)
$$

where $y_{1}=1 / 5$. The objective function value of the dual at $\boldsymbol{y}^{*}$ is

$$
\boldsymbol{b}^{\top} \boldsymbol{y}^{*}=\frac{1}{4} \log 30+\left(3 \log 3-2 \log 5+1-y_{1}\right) \varepsilon^{\prime}<\frac{1}{4} \log 30+\varepsilon^{\prime} \leqslant \frac{1}{4} \log 30+\varepsilon .
$$

So the family in 12$)$ is $(\varepsilon, 5)$-sufficient. This completes the proof. 